\title{
Ligand exchange-mediated activation and stabilization of a Re-based olefin metathesis catalyst by chlorinated alumina
}

Alessandro Gallo, Anthony Fong, Kai C. Szeto, Julia Rieb, Laurent Delevoye, Régis M. Gauvin,* Mostafa Taoufik,* Baron Peters,* Susannah L. Scott*

\author{
Corresponding authors: \\ regis.gauvin@ensc-lille.fr \\ taoufik@,cpe.fr \\ baronp@engineering.ucsb.edu \\ sscott@engineering.ucsb.edu
}

Figure S2 IR spectra of $\mathrm{Cl}-\mathrm{Al}_{2} \mathrm{O}_{3}$ after pyridine adsorption at RT followed by desorption for $20 \mathrm{~min}$ at (a) RT, (b) $200{ }^{\circ} \mathrm{C}$, and (c) $300{ }^{\circ} \mathrm{C}$

Figure S3 (a) ${ }^{1} \mathrm{H}$ MAS NMR spectrum, (b) ${ }^{1} \mathrm{H}-{ }^{27} \mathrm{Al}$ D-HMQC MAS NMR, and (c) ${ }^{27} \mathrm{Al}$ project, for $\mathrm{Cl}-\mathrm{Al}_{2} \mathrm{O}_{3}$

Figure S4 (a) ${ }^{1} \mathrm{H}$ MAS NMR spectrum, (b) ${ }^{1} \mathrm{H}-{ }^{27} \mathrm{Al}$ D-HMQC MAS NMR, $\quad$ S6 and (c) ${ }^{27} \mathrm{Al}$ project, for $\gamma-\mathrm{Al}_{2} \mathrm{O}_{3}$

$\begin{array}{llll}\text { Figure S5 } & { }_{1}^{1} \mathrm{H} \text { MAS NMR spectrum, and (b) }{ }^{1} \mathrm{H}-{ }^{1} \mathrm{H} \text { DQ-SQ MAS spectrum, } & \text { S7 }\end{array}$ for $\mathrm{MTO} / \mathrm{Cl}-\mathrm{Al}_{2} \mathrm{O}_{3}$

Figure S6 (a) ${ }^{1} \mathrm{H}$ MAS NMR, and (b) ${ }^{1} \mathrm{H}-{ }^{1} \mathrm{H}$ DQ-SQ MAS NMR, for MTO $/ \gamma-\quad$ S8 $\mathrm{Al}_{2} \mathrm{O}_{3}$

Figure S7 Comparison of ${ }^{13} \mathrm{C} \mathrm{CP}-\mathrm{MAS} \mathrm{NMR}$ for (a) $\mathrm{MTO} / \mathrm{Cl}-\mathrm{Al}_{2} \mathrm{O}_{3}$, and (b) S9

Figure S8 Deconvolution of ${ }^{13} \mathrm{C} \mathrm{CP-MAS} \mathrm{NMR} \mathrm{for} \mathrm{MTO/Cl-} \mathrm{Al}_{2} \mathrm{O}_{3} \quad \mathrm{~S} 9$

Figure S9 Comparison of Re $L_{\mathrm{I}}$-edge XANES for MTO diluted in BN, $\quad \mathrm{S} 10$ $\mathrm{MTO} / \gamma-\mathrm{Al}_{2} \mathrm{O}_{3}$, and $\mathrm{MTO} / \mathrm{Cl}-\mathrm{Al}_{2} \mathrm{O}_{3}$

Figure S10 Comparison of Re $L_{\mathrm{III}}$-edge XANES for MTO diluted in BN, $\mathrm{S} 10$ $\mathrm{MTO} / \gamma-\mathrm{Al}_{2} \mathrm{O}_{3}$, and $\mathrm{MTO} / \mathrm{Cl}-\mathrm{Al}_{2} \mathrm{O}_{3}$

Figure S11 Re $L_{\mathrm{III}}$-edge EXAFS and curvefit for $\mathrm{MTO} / \gamma-\mathrm{Al}_{2} \mathrm{O}_{3}, k^{3}$-weighted, in $\quad \mathrm{S} 11$ (a) $k$-space, and (b) $R$-space

Figure S12 Re $L_{\mathrm{III}}$-edge EXAFS and curvefit for $\mathrm{MTO} / \mathrm{Cl}-\mathrm{Al}_{2} \mathrm{O}_{3}, k^{3}$-weighted, $\quad \mathrm{S} 11$ in $k$-space

Figure S13 (a) ${ }^{17} \mathrm{O}$ MAS NMR spectrum of ${ }^{17} \mathrm{O}$-labelled MTO, and ${ }^{17} \mathrm{O}$ echo MAS NMR spectra of (b) ${ }^{17} \mathrm{O}$-enriched $\gamma-\mathrm{Al}_{2} \mathrm{O}_{3}$, and (c) MTO-Cl$\mathrm{Al}_{2} \mathrm{O}_{3}$

Figure $\mathrm{S14}$ Evolution of propene metathesis activity for $\mathrm{MTO} / \mathrm{Cl}-\mathrm{Al}_{2} \mathrm{O}_{3}$ (green) and $\mathrm{MTO} / \gamma-\mathrm{Al}_{2} \mathrm{O}_{3}$ (black), in a flow reactor 
Figure S15 DFT cluster model for $\mathrm{Al}_{2} \mathrm{Cl}_{6} . \quad \mathrm{S} 13$

$\begin{array}{lll}\text { Table S1 Key inter-atomic distances and bond angles computed for } \mathrm{Al}_{2} \mathrm{Cl}_{6} & \mathrm{~S} 14\end{array}$

$\begin{array}{lll}\text { Table S2 } & \text { DFT-optimized bond lengths for MTO interacting with } \mathrm{Al}_{2} \mathrm{Cl}_{6} & \mathrm{~S} 14\end{array}$

$\begin{array}{lll}\text { Scheme S2 DFT-optimized structures for additional adducts of MTO with } & \text { S14 }\end{array}$ $\mathrm{Al}_{2} \mathrm{Cl}_{6}$

Table S3 Calculated ${ }^{1} \mathrm{H}$ chemical shifts (ppm) for $\mathrm{MTO} / \mathrm{Cl}^{-}-\mathrm{Al}_{2} \mathrm{O}_{3}$ and related $\quad \mathrm{S} 15$

Table S4 Calculated ${ }^{13} \mathrm{C}$ chemical shifts (ppm) for $\mathrm{MTO} / \mathrm{Al}_{2} \mathrm{Cl}_{6}$ models, and $\quad \mathrm{S} 15$ related carbene tautomers

$\begin{array}{lll}\text { Scheme S3 Computed structures for additional carbene tautomers of MTO } & \text { S16 }\end{array}$ interacting with $\mathrm{Al}_{2} \mathrm{Cl}_{6}$

Figure S16 Comparison of ${ }^{13} \mathrm{C}$ CP-MAS NMR for (a) MTO/Cl- $\mathrm{Al}_{2} \mathrm{O}_{3}$, and (b) $\quad \mathrm{S} 16$ $\mathrm{Cl}-\mathrm{Al}_{2} \mathrm{O}_{3}$, after $1 \mathrm{~h}$ reaction with $\mathrm{CH}_{3}{ }^{13} \mathrm{CH}=\mathrm{CH}_{2}$ at $0{ }^{\circ} \mathrm{C}$.

$\begin{array}{lll}\text { Scheme S4 } & \text { DFT-optimized energies and computed }{ }^{13} \mathrm{C} \text { NMR chemical shifts } & \text { S17 }\end{array}$ for rhenacyclobutanes derived from a terminal carbene

$\begin{array}{llll}\text { Table S5 Calculated }{ }^{13} \mathrm{C} \text { chemical shifts for molecular organorhenium(VII) } & \text { S17 }\end{array}$ compounds

Figure S17 Calibration curve for calculated ${ }^{13} \mathrm{C}$ NMR chemical shifts in $\quad$ S18 organorhenium(VII) compounds

$\begin{array}{lll}\text { Table S6 Calculated }{ }^{1} \mathrm{H} \text { chemical shifts for molecular organorhenium(VII) } & \text { S18 }\end{array}$ compounds

Figure S18 Calibration curve for calculated ${ }^{1} \mathrm{H}$ NMR chemical shifts in Re(VII) $\quad$ S19 compounds

Table S7 DFT-optimized bond lengths for carbene tautomers of MTO S19 interacting with $\mathrm{Al}_{2} \mathrm{Cl}_{6}$

Table S8 Cartesian coordinates and total energies for all computed structures $\quad$ S20 $\begin{array}{ll}\text { Sample Gaussian input file } & \text { S24 }\end{array}$ $\begin{array}{ll}\text { Additional references } & \text { S25 }\end{array}$ 


\section{Sample calculation of initial turnover frequency from rate constant}

For MTO/Cl- $\mathrm{Al}_{2} \mathrm{O}_{3}, \quad \mathrm{k}=98 \mathrm{~s}^{-1}(\mathrm{~g} \mathrm{Re})^{-1}(186.2 \mathrm{~g} \mathrm{Re} / \mathrm{mol} \mathrm{Re})=18,250 \mathrm{~s}^{-1}(\mathrm{~mol} \mathrm{Re})^{-1}$ $\mathrm{m}_{\mathrm{Re}}=10 \mathrm{mg} \times 0.024 \mathrm{~g} \mathrm{Re} / \mathrm{g}$ cat $=0.24 \mathrm{mg} R \mathrm{Re}=2.4 \times 10^{-4} \mathrm{~g} \mathrm{Re}$ $\mathrm{n}_{\mathrm{Re}}=2.4 \times 10^{-4} \mathrm{~g} \mathrm{Re} /\left(186.2 \mathrm{~g} \mathrm{~mol}^{-1}\right)=1.29 \times 10^{-6} \mathrm{~mol} \mathrm{Re}=1.29 \mu \mathrm{mol} \mathrm{Re}$ $\mathrm{P}(\mathrm{C} 3)=67$ mbar, $\mathrm{T}=273 \mathrm{~K}, \mathrm{~V}=0.12 \mathrm{~L}$

Initial rate of propene conversion

$$
\begin{aligned}
& \mathrm{r}=\mathrm{k} \mathrm{n}_{\mathrm{Re}} \mathrm{P}(\mathrm{C} 3)=\left(18,250 \mathrm{~s}^{-1} \mathrm{~mol}^{-1}\right)\left(1.3 \times 10^{-6} \mathrm{~mol}\right)(0.067 \mathrm{bar}) \\
& =0.00158 \mathrm{bar} \mathrm{s}^{-1} \\
& \mathrm{r}=\left(0.00158 \mathrm{bar} \mathrm{s}^{-1}\right)(1.013 \mathrm{~atm} / \mathrm{bar})(0.12 \mathrm{~L}) /(0.082 \mathrm{~L} \cdot \mathrm{atm} / \mathrm{K} \cdot \mathrm{mol})(273 \mathrm{~K}) \\
& =8.7 \times 10^{-6} \mathrm{~mol} \mathrm{C} 3 \mathrm{~s}^{-1}=8.7 \mu \mathrm{mol} \mathrm{C} 3 \mathrm{~s}^{-1}
\end{aligned}
$$

conversion to instantaneous TOF $(\mathrm{t}=0 \mathrm{~s})$

$$
\mathrm{TOF}=\text { rate } / \mathrm{mol} \operatorname{Re}=\left(8.7 \mu \mathrm{mol} \mathrm{C} 3 \mathrm{~s}^{-1}\right) /(1.29 \mu \mathrm{mol} \operatorname{Re})=6.6 \mathrm{~s}^{-1}
$$

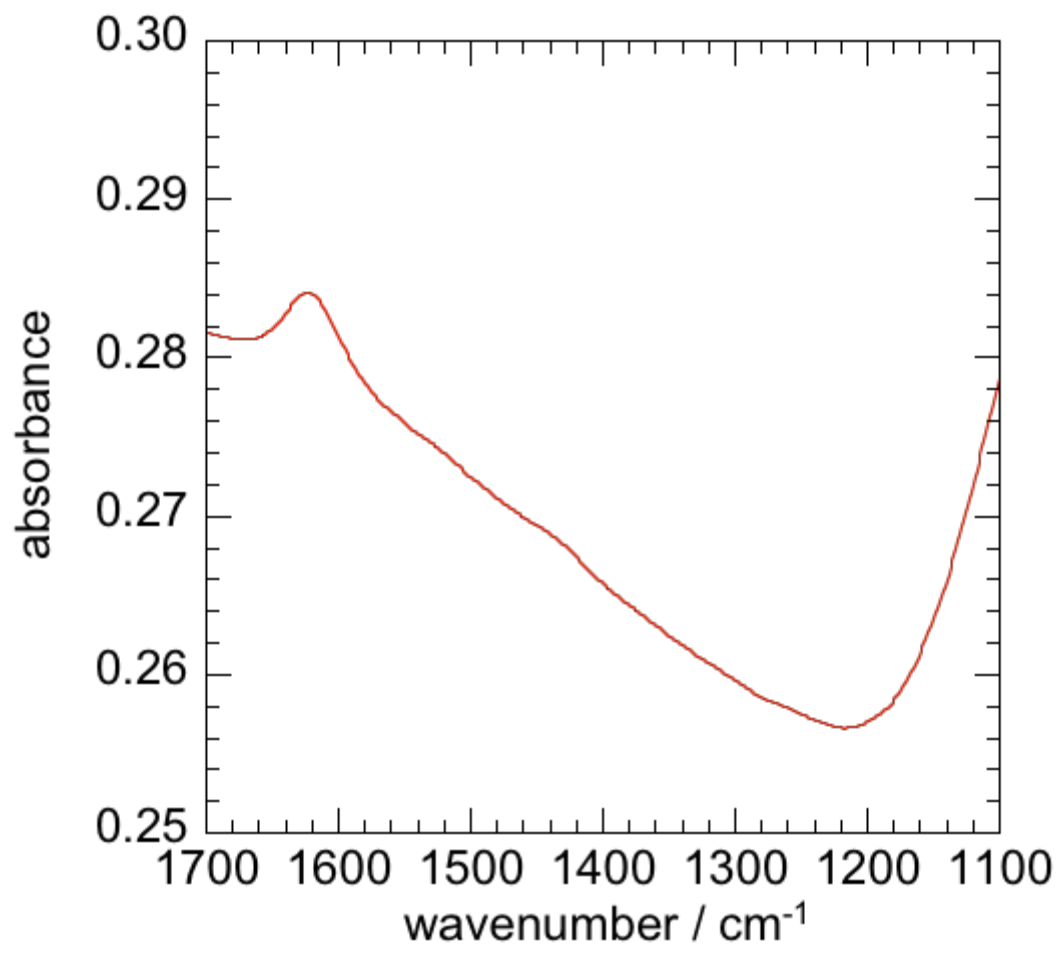

Figure S1. Mid-IR spectrum of $\mathrm{Cl}-\mathrm{Al}_{2} \mathrm{O}_{3}$, showing a small peak for adsorbed $\mathrm{H}_{2} \mathrm{O}\left(1630 \mathrm{~cm}^{-1}\right)$ and the absence of $\mathrm{AlCl}_{3}\left(1380 \mathrm{~cm}^{-1}\right)$. 


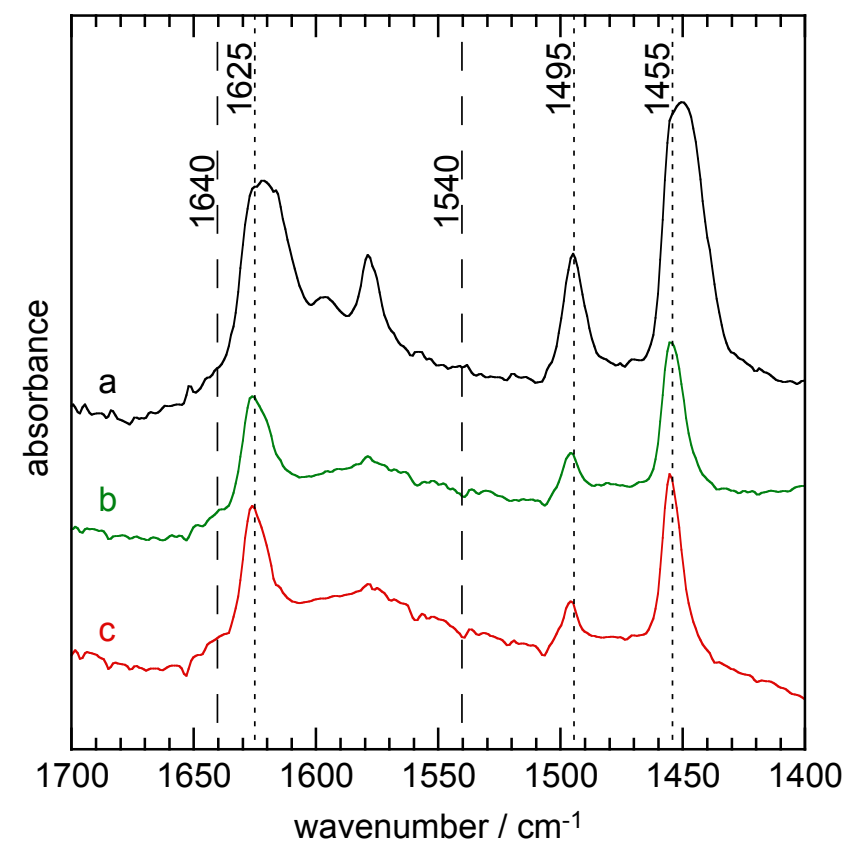

Figure S2. IR spectra of $\mathrm{Cl}-\mathrm{Al}_{2} \mathrm{O}_{3}(4.0 \mathrm{wt} \% \mathrm{Cl})$ after pyridine adsorption at $\mathrm{RT}$ followed by desorption for $20 \mathrm{~min}$ at (a) RT (black), (b) $200{ }^{\circ} \mathrm{C}$ (green), and (c) $300{ }^{\circ} \mathrm{C}$ (red). The spectra show evidence for strong Lewis acidity, in the persistence of bands at 1625,1495 and $1455 \mathrm{~cm}^{-1}$ attributed to adsorbed pyridine at elevated temperatures. ${ }^{1}$ The lack of significant Brønsted acidity is indicated by the absence of bands at 1640 and $1540 \mathrm{~cm}^{-1}$ for adsorbed pyridinium. 


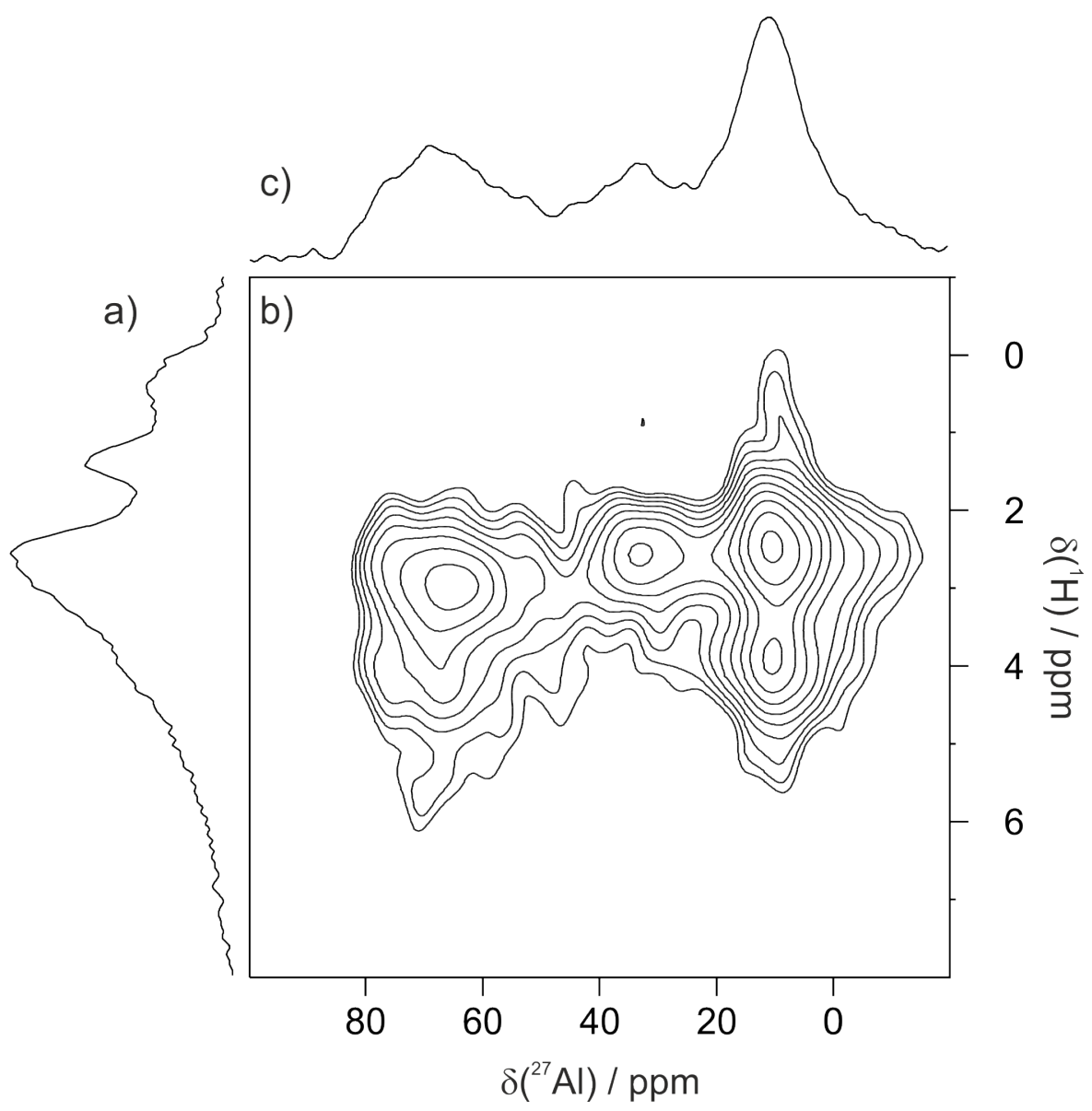

Figure S3. (a) ${ }^{1} \mathrm{H}$ MAS NMR, (b) ${ }^{1} \mathrm{H}^{27} \mathrm{Al}$ D-HMQC MAS NMR (18.8 T, spinning speed 20 $\mathrm{kHz}$, recycle delay $2 \mathrm{~s}, 500 \mu \mathrm{s})$, and (c) ${ }^{27} \mathrm{Al}$ projection, for $\mathrm{Cl}-\mathrm{Al}_{2} \mathrm{O}_{3}(4.0 \mathrm{wt} \% \mathrm{Cl})$ evacuated at $450{ }^{\circ} \mathrm{C}$. 


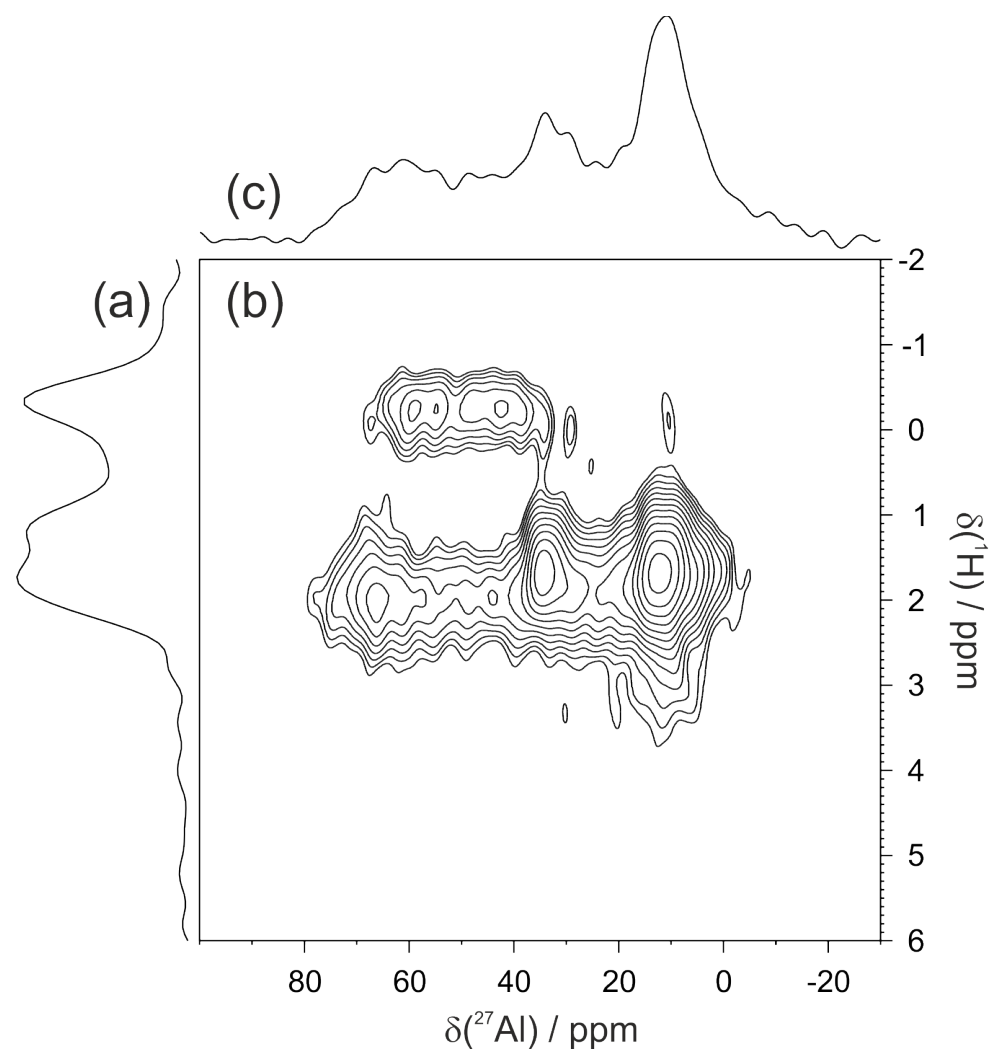

Figure S4. (a) ${ }^{1} \mathrm{H}$ MAS NMR, (b) ${ }^{1} \mathrm{H}_{-}{ }^{27} \mathrm{Al} \mathrm{D}-\mathrm{HMQC}$ MAS NMR (18.8 T, spinning speed 20 $\mathrm{kHz}$, recycle delay $2 \mathrm{~s}, 500 \mu \mathrm{s}$ ), and (c) ${ }^{27} \mathrm{Al}$ projection, for $\gamma-\mathrm{Al}_{2} \mathrm{O}_{3}$ evacuated at $450^{\circ} \mathrm{C}$. 


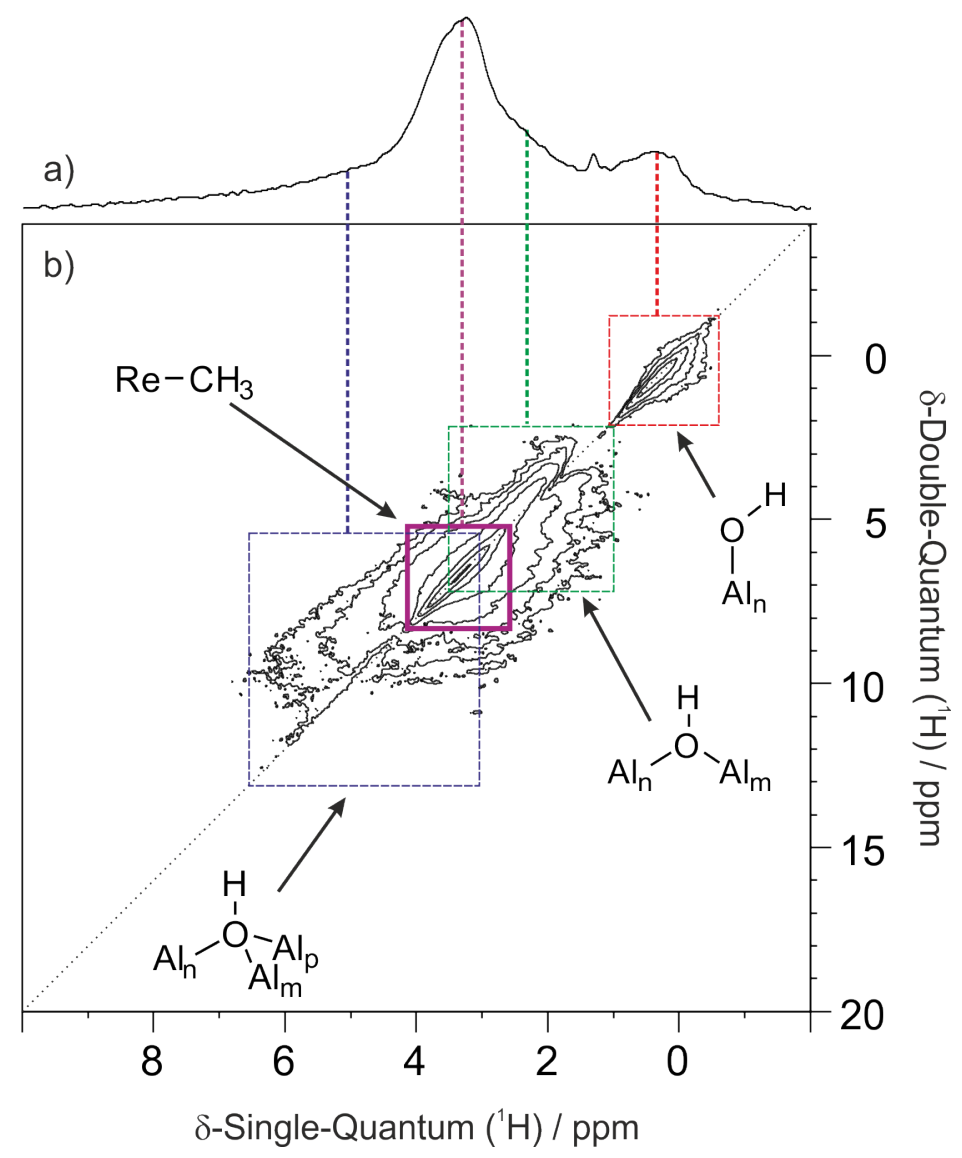

Figure S5. (a) ${ }^{1} \mathrm{H}$ MAS NMR spectrum, and (b) ${ }^{1} \mathrm{H}-{ }^{1} \mathrm{H}$ DQ-SQ MAS spectrum, for MTO/Cl$\mathrm{Al}_{2} \mathrm{O}_{3}(18.8 \mathrm{~T}$, spinning speed $20 \mathrm{kHz}$, recycle delay $2 \mathrm{~s}$, recoupling time $1 \mathrm{~ms})$. 


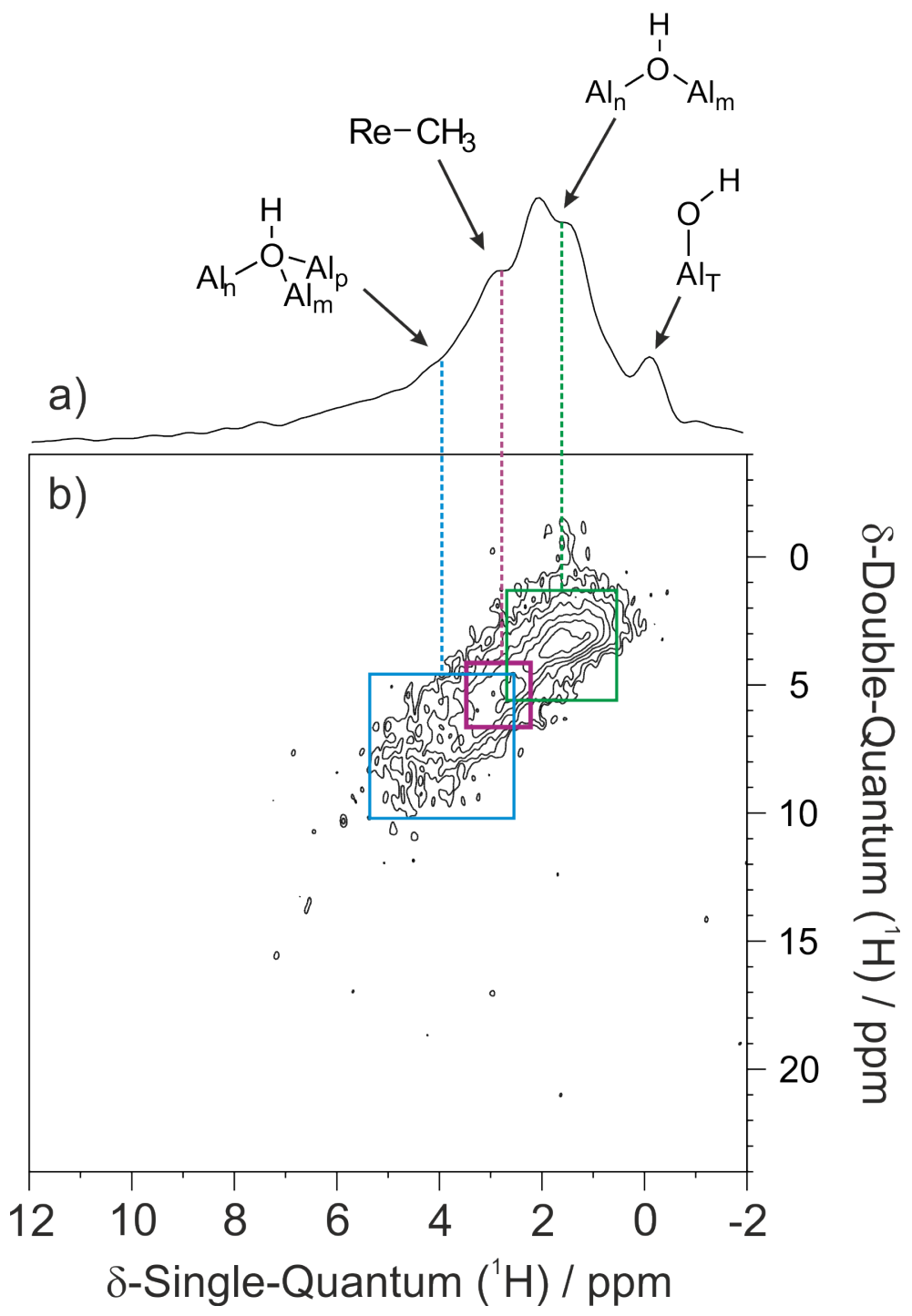

Figure S6. (a) ${ }^{1} \mathrm{H}$ MAS NMR, and (b) ${ }^{1} \mathrm{H}-{ }^{1} \mathrm{H}$ DQ-SQ MAS NMR (18.8 T, spinning speed 20 $\mathrm{kHz}$, recycle delay $2 \mathrm{~s}$, recoupling time $1 \mathrm{~ms})$, for $\mathrm{MTO} / \gamma-\mathrm{Al}_{2} \mathrm{O}_{3}(2.3 \mathrm{wt} . \% \mathrm{Re})$. 


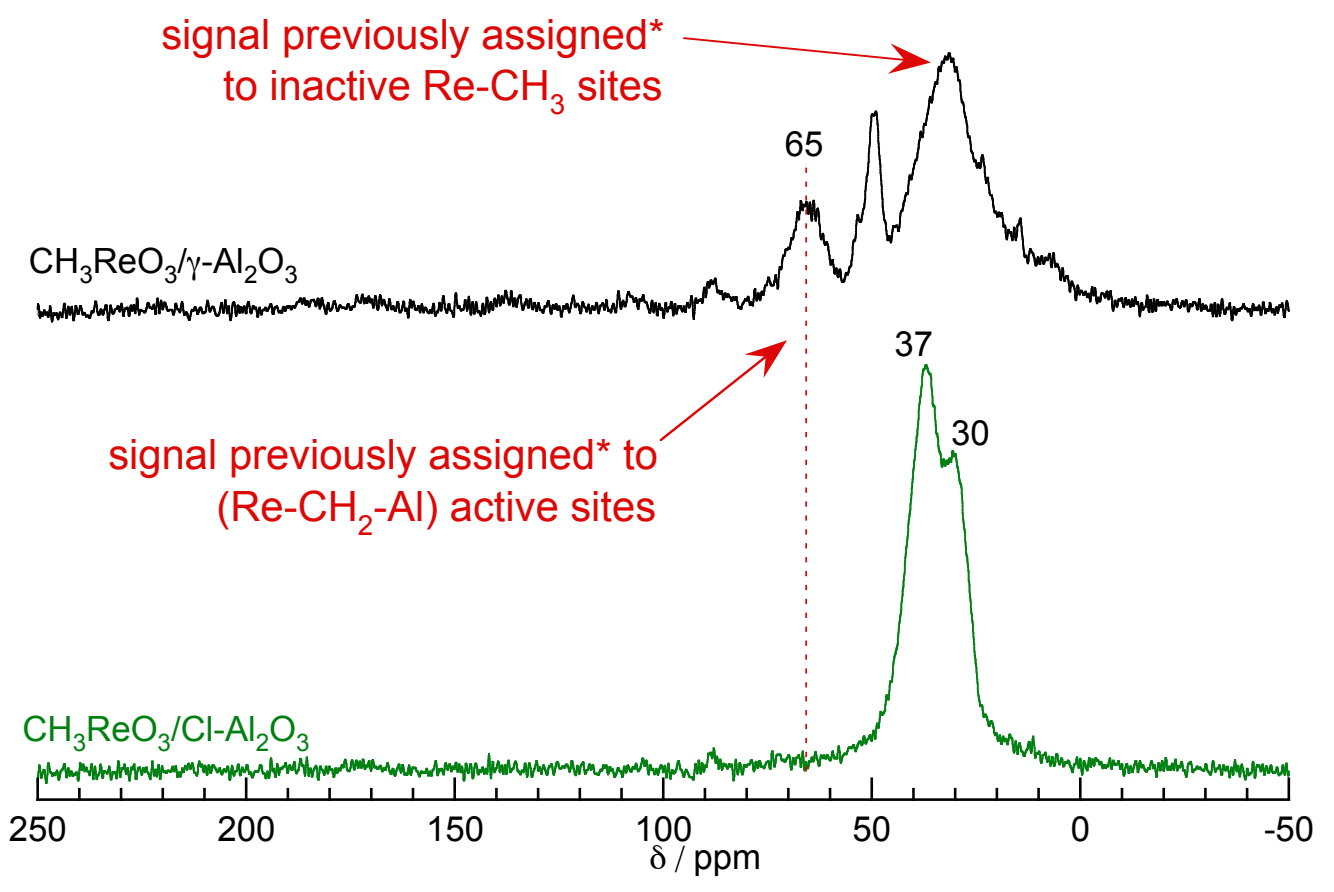

Figure S7. Comparison of ${ }^{13} \mathrm{C}$ CP-MAS NMR for (a) $\mathrm{MTO} / \mathrm{Cl}-\mathrm{Al}_{2} \mathrm{O}_{3}(2.4 \mathrm{wt} \% \mathrm{Re}, 4.0 \mathrm{wt} \% \mathrm{Cl}$, green), and (b) $\mathrm{MTO} / \gamma-\mathrm{Al}_{2} \mathrm{O}_{3}(2.5 \mathrm{wt} \% \mathrm{Re}$, black). $7.07 \mathrm{~T}$, spinning speed $10 \mathrm{kHz}$, showing signals $\left(^{*}\right)$ with earlier assignments. ${ }^{2}$

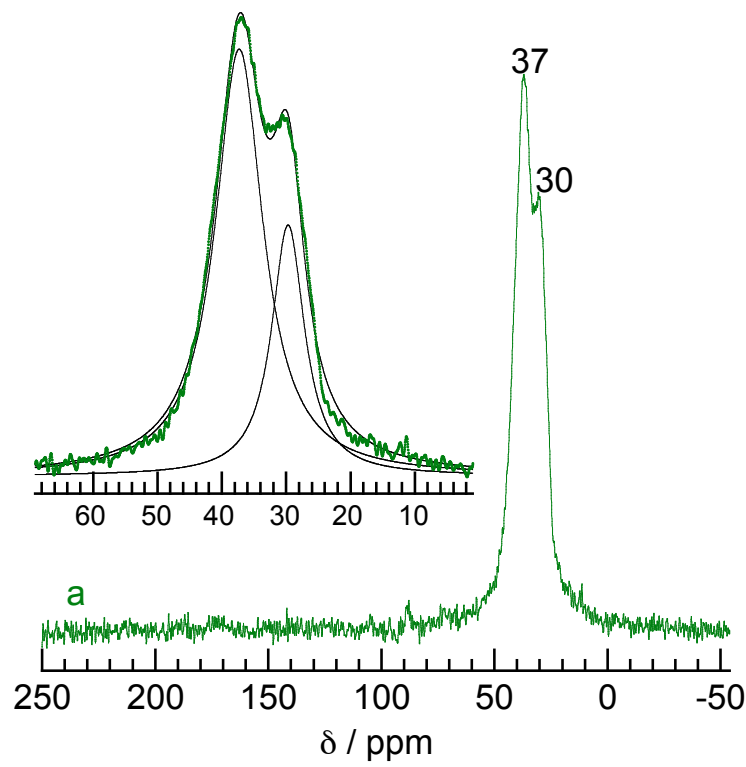

Figure S8. Deconvolution of ${ }^{13} \mathrm{C}$ CP-MAS NMR for $\mathrm{MTO} / \mathrm{Cl}^{-\mathrm{Al}_{2} \mathrm{O}_{3}}(2.4 \mathrm{wt} \% \mathrm{Re}, 4.0 \mathrm{wt} \% \mathrm{Cl}$, $7.05 \mathrm{~T}$, spinning rate $10 \mathrm{kHz}$ ) using two Lorentzian components. 


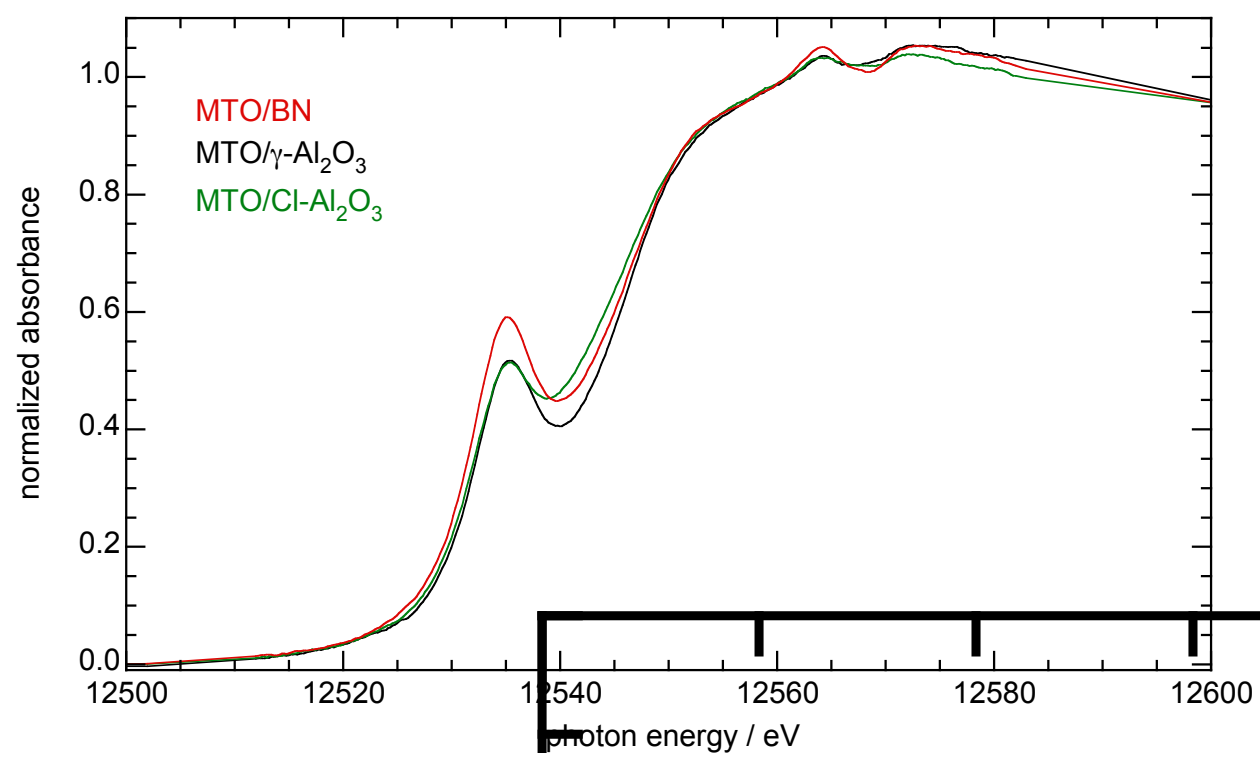

Figure S9. Comparison of Re $L_{\mathrm{I}}$-edge XANES for MTO diluted in $\mathrm{BN}$ (red), MTO $/ \gamma-\mathrm{Al}_{2} \mathrm{O}_{3}(2.4$ $\mathrm{wt} \% \mathrm{Re}$, black), and $\mathrm{MTO} / \mathrm{Cl}-\mathrm{Al}_{2} \mathrm{O}_{3}(2.4 \mathrm{wt} \% \mathrm{Re}, 4.0 \mathrm{wt} \% \mathrm{Cl}$, green). The edge position, 12,539 $\mathrm{eV}$ in all three spectra, is consistent with $\mathrm{Re}(\mathrm{VII})$. The prominent pre-edge peak, due to the dipole $2 s \rightarrow 5 d$ transition, appears at $12,526 \mathrm{eV}$ in all three spectra. It indicates noncentrosymmetric geometry at Re that allows $5 p-6 d$ hybridization. A similar pre-edge feature was detected for $\mathrm{Re}_{2} \mathrm{O}_{7}$ and $\mathrm{NH}_{4} \mathrm{ReO}_{4}{ }^{3,4}$ The intensity decreases upon grafting MTO onto either $\gamma$ $\mathrm{Al}_{2} \mathrm{O}_{3}$ or $\mathrm{Cl}-\mathrm{Al}_{2} \mathrm{O}_{3}$, suggesting a decrease in symmetry.

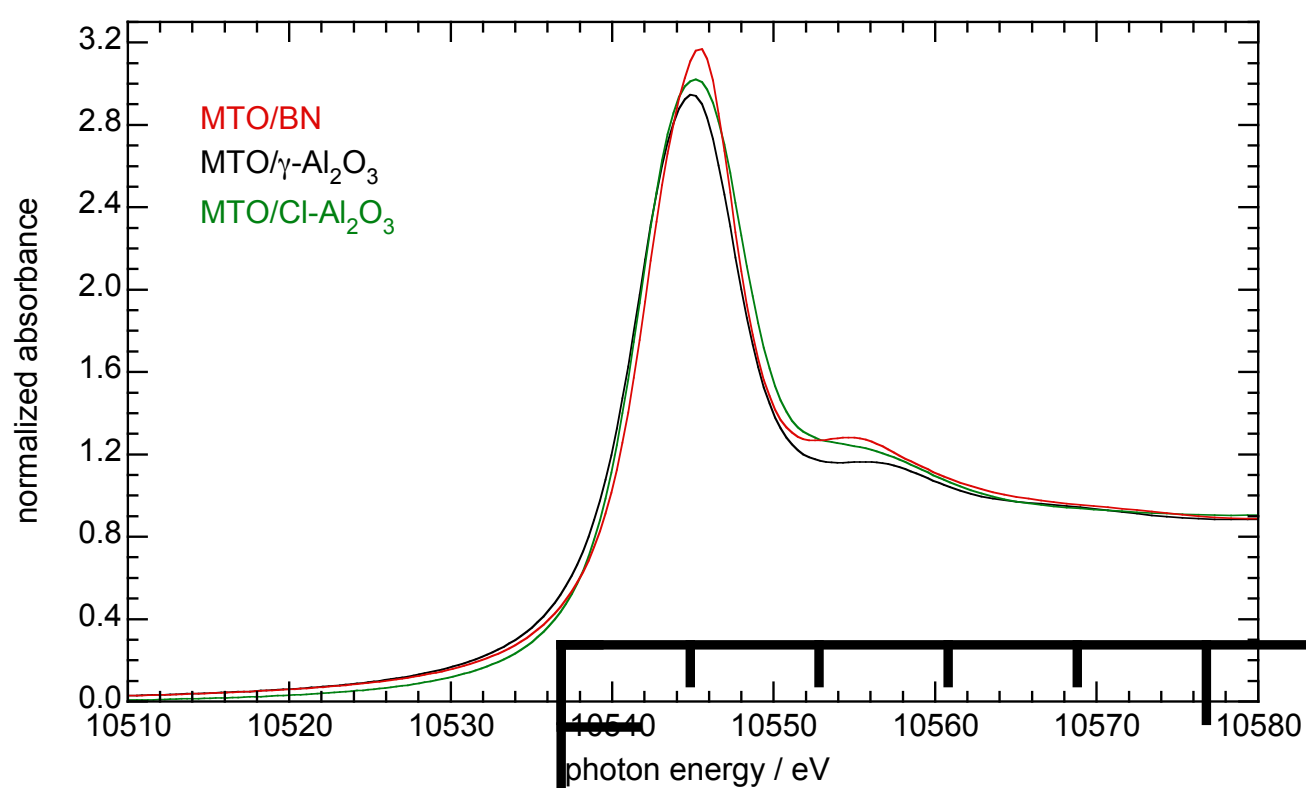

Figure S10. Comparison of Re $L_{\mathrm{III}}$-edge XANES for MTO diluted in $\mathrm{BN}$ (red), $\mathrm{MTO} / \gamma-\mathrm{Al}_{2} \mathrm{O}_{3}$ (2.4 $\mathrm{wt} \% \mathrm{Re}$, black), and $\mathrm{MTO} / \mathrm{Cl}^{-\mathrm{Al}_{2} \mathrm{O}_{3}}(2.4 \mathrm{wt} \% \mathrm{Re}, 4.0 \mathrm{wt} \% \mathrm{Cl}$, green). Each shows a pronounced white line, similar to $\mathrm{Re}_{2} \mathrm{O}_{7}$ and $\mathrm{NH}_{4} \mathrm{ReO}_{4},{ }^{3}$ characteristic of a high Re oxidation state. The edge position, $10,542 \mathrm{eV}$, is the same for all three spectra, and is consistent with $\operatorname{Re}(\mathrm{VII})$. 

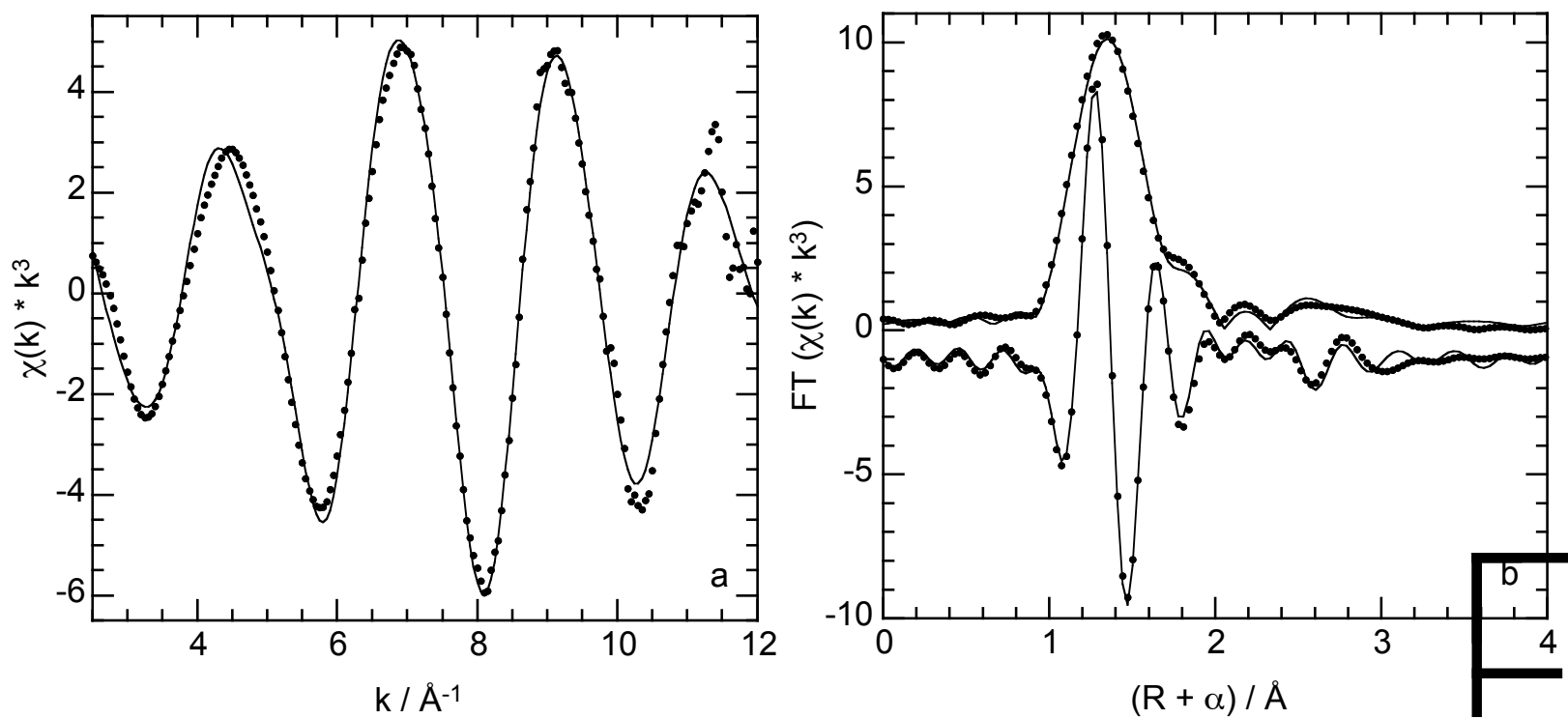

Figure S11. Re $L_{\mathrm{III}}$-edge EXAFS (circles) for $\mathrm{MTO} / \gamma-\mathrm{Al}_{2} \mathrm{O}_{3}(2.4 \mathrm{wt} \% \mathrm{Re}), k^{3}$-weighted, in (a) $k$ space, and (b) non-phase-corrected $R$-space. Parameters for the curve-fit (lines) are given in Table 2 (see main text).

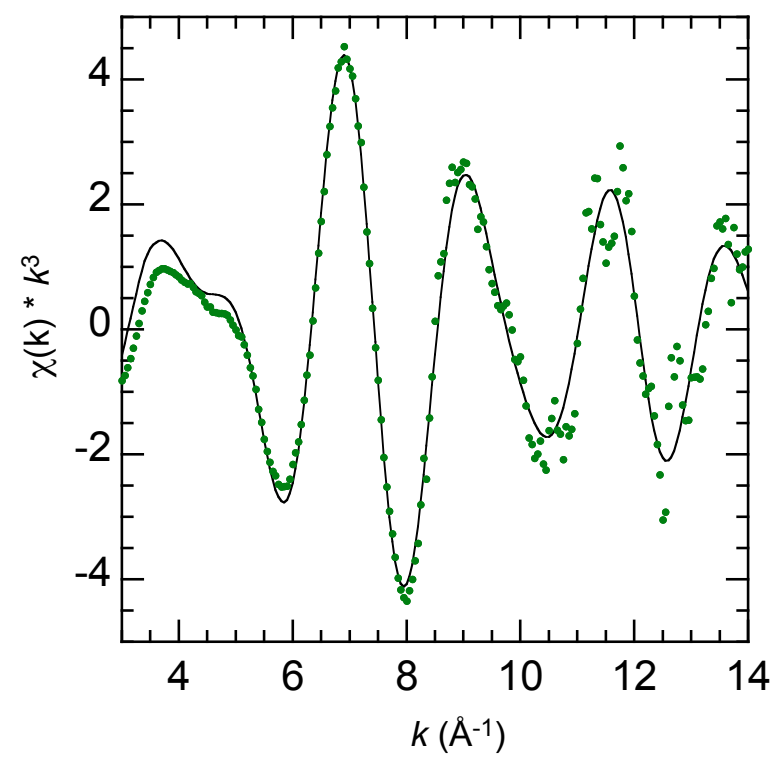

Figure S12. Re $L_{\mathrm{III}}$-edge EXAFS (circles) for $\mathrm{MTO} / \mathrm{Cl}-\mathrm{Al}_{2} \mathrm{O}_{3}(2.4 \mathrm{wt} \% \mathrm{Re}, 4.0 \mathrm{wt} \% \mathrm{Cl}), k^{3}$ weighted, in $k$-space, showing curve-fit (line) for parameters given in Table 2 (see main text). 


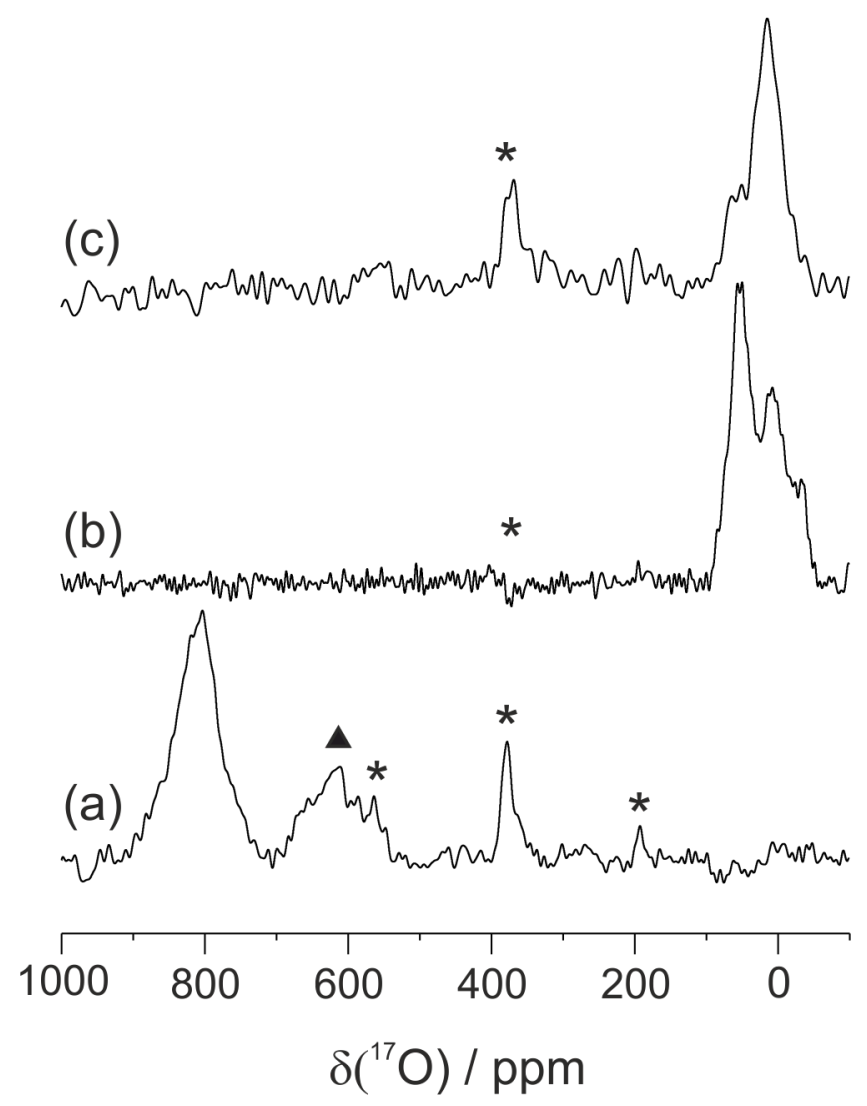

Figure S13. (a) ${ }^{17} \mathrm{O}$ MAS NMR spectrum of ${ }^{17} \mathrm{O}$-labelled MTO (6300 transients), and ${ }^{17} \mathrm{O}$ echo MAS NMR spectra of (b) ${ }^{17} \mathrm{O}$-enriched $\gamma$ - $\mathrm{Al}_{2} \mathrm{O}_{3}$ (72 k transients) and (c) MTO-Cl- $\mathrm{Al}_{2} \mathrm{O}_{3}(110 \mathrm{k}$ transients). General conditions: $18.8 \mathrm{~T}$, spinning speed $20 \mathrm{kHz}$, relaxation delay $1 \mathrm{~s}$. * denotes rotor $\left(\mathrm{ZrO}_{2}\right)$ signals and corresponding spinning side-bands; dark triangle indicates spinning side-band of MTO signal. 


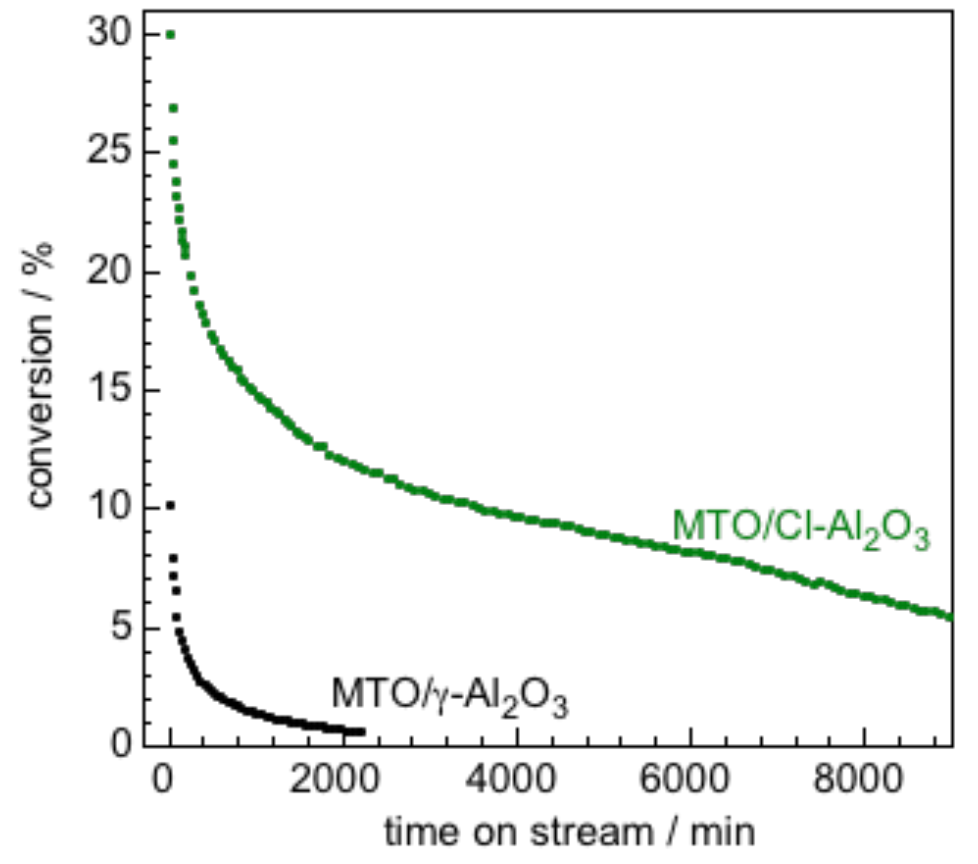

Figure S14. Evolution of propene metathesis activity for $\mathrm{MTO} / \mathrm{Cl}-\mathrm{Al}_{2} \mathrm{O}_{3}$ (green) and $\mathrm{MTO} / \gamma$ $\mathrm{Al}_{2} \mathrm{O}_{3}$ (black), in a flow reactor. Conditions: $10^{\circ} \mathrm{C}, 1 \mathrm{bar}, 1.92 \mathrm{~mol} \mathrm{C}_{3} \mathrm{H}_{6} \cdot \mathrm{s}^{-1} \cdot(\mathrm{mol} \mathrm{Re})^{-1}$.

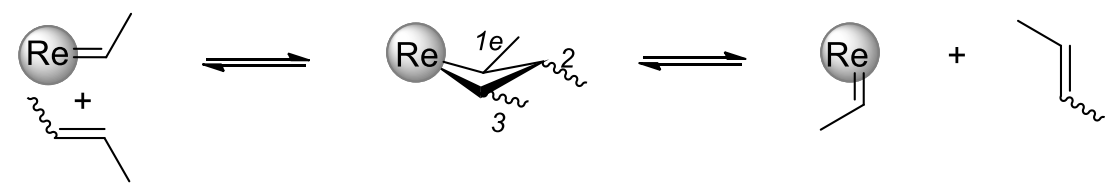

Scheme S1. Isomerization of 2-butenes by degenerate metathesis.

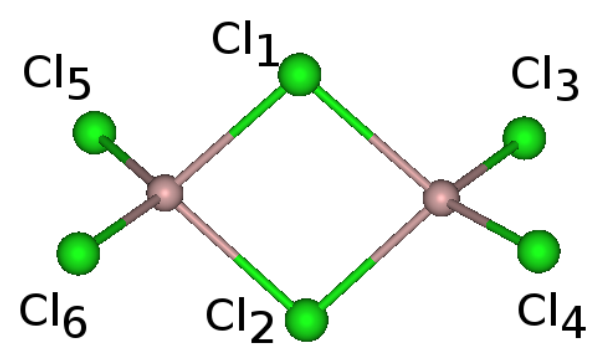

Figure S15. DFT cluster model for $\mathrm{Al}_{2} \mathrm{Cl}_{6}$. Color scheme: $\mathrm{Cl}$, green; $\mathrm{Al}$, purple. 
Table S1. Key inter-atomic distances $(\AA)$ and bond angles $\left(^{\circ}\right)$ computed for the $\mathrm{Al}_{2} \mathrm{Cl}_{6}$ cluster

\begin{tabular}{cccc}
\hline Distance & Value & Angle & Value \\
\hline Al-Cl1 & 2.31 & Al-Cl1-Al & 93 \\
Al-Cl2 & 2.31 & Al-Cl2-Al & 93 \\
Al-Cl3 & 2.09 & Cl1-Al-Cl3 & 107 \\
Al-C14 & 2.12 & Cl1-Al-Cl4 & 116 \\
\hline
\end{tabular}

Table S2. DFT-optimized bond lengths ( $\AA$ ) for MTO interacting with $\mathrm{Al}_{2} \mathrm{Cl}_{6}$

\begin{tabular}{lcccccc}
\hline & MTO & Ia & IIa & IIIa & IVa & Va \\
\hline Re-O1 & 1.69 & 1.78 & 1.94 & 1.73 & 1.85 & 1.76 \\
Re-O2 & 1.69 & 1.78 & 1.67 & 1.68 & 1.67 & 1.67 \\
Re-O3 & 1.69 & 1.66 & 1.66 & 1.68 & 1.67 & 1.67 \\
Re-Cl & -- & 2.31 & 2.41 & & 2.49 & \\
Re-C & -- & 2.14 & 2.13 & 2.07 & 2.14 & 2.07 \\
\hline
\end{tabular}
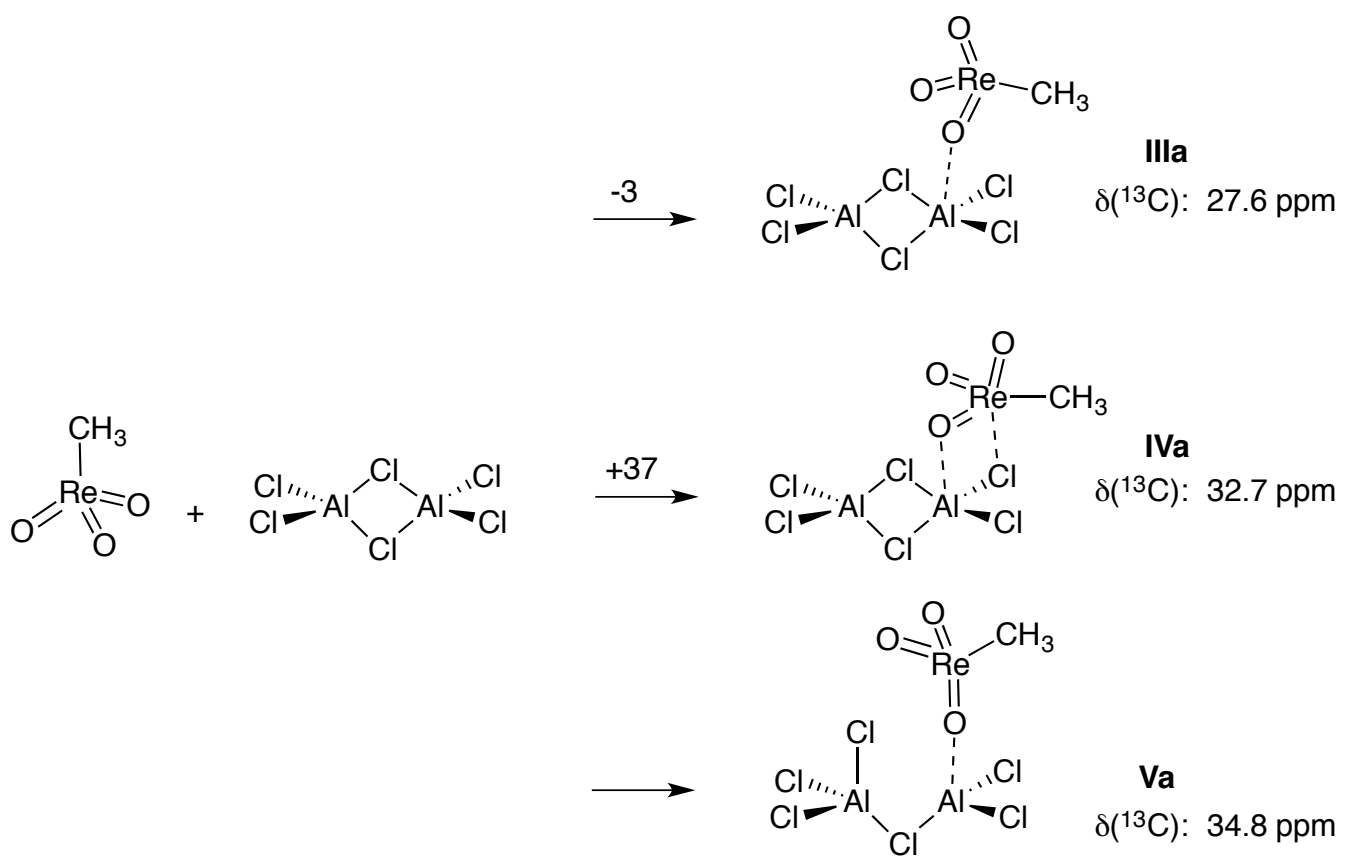

Scheme S2. DFT-optimized structures for additional adducts of $\mathrm{MTO}$ with $\mathrm{Al}_{2} \mathrm{Cl}_{6}$, with computed free energies at $298 \mathrm{~K}$ in $\mathrm{kJ} / \mathrm{mol}$. 
Table S3. Calculated ${ }^{1} \mathrm{H}$ chemical shifts (ppm) for $\mathrm{MTO} / \mathrm{Cl}-\mathrm{Al}_{2} \mathrm{O}_{3}$ and related carbene tautomers

\begin{tabular}{|c|c|c|}
\hline Species & Calculated isotropic shielding & Calculated chemical shift \\
\hline Ia & 29.34 & 2.95 \\
\hline IIa & 29.68 & 2.66 \\
\hline IIIa & 29.87 & 2.51 \\
\hline IVa & 30.04 & 2.36 \\
\hline Va & 29.81 & 2.56 \\
\hline \multirow[t]{2}{*}{ Ib } & 16.17 & $13.57\left(\mathrm{CH}_{2}\right)$ \\
\hline & 30.18 & $2.25(\mathrm{OH})$ \\
\hline \multirow[t]{2}{*}{ IIb } & 17.14 & $13.20\left(\mathrm{CH}_{2}\right)$ \\
\hline & 29.71 & $2.63(\mathrm{OH})$ \\
\hline \multirow[t]{2}{*}{ IIIb } & 19.08 & $11.57\left(\mathrm{CH}_{2}\right)$ \\
\hline & 25.33 & $6.32(\mathrm{OH})$ \\
\hline \multirow[t]{2}{*}{$\mathbf{V b}$} & 18.34 & $12.19\left(\mathrm{CH}_{2}\right)$ \\
\hline & 24.09 & $7.36(\mathrm{OH})$ \\
\hline \multirow[t]{2}{*}{ Ic } & $26.71,29.13$ & $5.16,3.13\left(\mathrm{CH}_{2}\right)$ \\
\hline & 40.07 & $2.34(\mathrm{OH})$ \\
\hline \multirow[t]{2}{*}{ IIc } & $17.62,15.70$ & $12.80,14.42\left(\mathrm{CH}_{2}\right)$ \\
\hline & 22.68 & $8.55(\mathrm{OH})$ \\
\hline \multirow[t]{2}{*}{ Id } & 19.35 & $11.35\left(\mathrm{CH}_{2}\right)$ \\
\hline & 22.57 & $8.64(\mathrm{OH})$ \\
\hline
\end{tabular}

Table S4. Calculated ${ }^{13} \mathrm{C}$ chemical shifts (ppm) for $\mathrm{MTO} / \mathrm{Al}_{2} \mathrm{Cl}_{6}$ models, and related carbene tautomers

\begin{tabular}{ccc}
\hline model & $\begin{array}{c}\text { Calculated } \\
\text { isotropic shielding }\end{array}$ & $\begin{array}{c}\text { Calculated } \\
\text { chemical shift }\end{array}$ \\
\hline Ia & 147.1 & 42.7 \\
IIa & 149.8 & 40.1 \\
IIIa & 163.1 & 27.6 \\
IVa & 157.7 & 32.7 \\
Va & 155.4 & 34.8 \\
Ib & -114.1 & 290 \\
IIb & -105.9 & 282 \\
IIIb & -62.1 & 241 \\
Vb & -68.7 & 247 \\
Ic & 104.8 & 82 \\
IIc & -107.7 & 284 \\
Id & -58.5 & 237 \\
\hline
\end{tabular}


<smiles>CP1(=O)O[Al](Cl)(Cl)O[Al](Cl)(Cl)O1</smiles>

la<smiles></smiles>

Ila

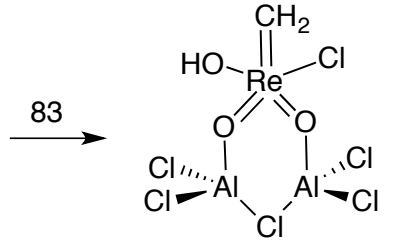

Id

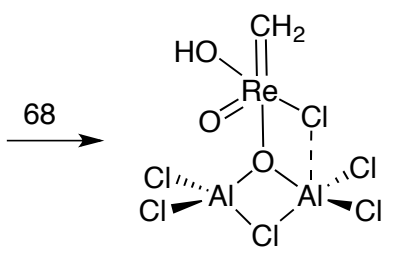

IIc

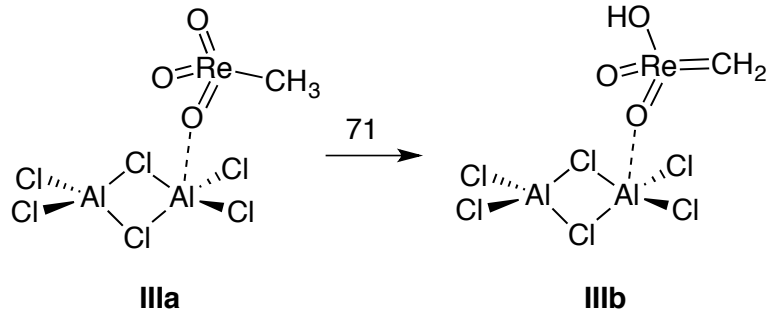<smiles>C[R](=O)(O)O[Al](Cl)(Cl)[Al](Cl)(Cl)Cl</smiles>

Va<smiles></smiles>

Vb

Scheme S3. Computed structures for additional carbene tautomers of MTO interacting with $\mathrm{Al}_{2} \mathrm{Cl}_{6}$, with computed free energies at $298 \mathrm{~K}$ in $\mathrm{kJ} / \mathrm{mol}$.

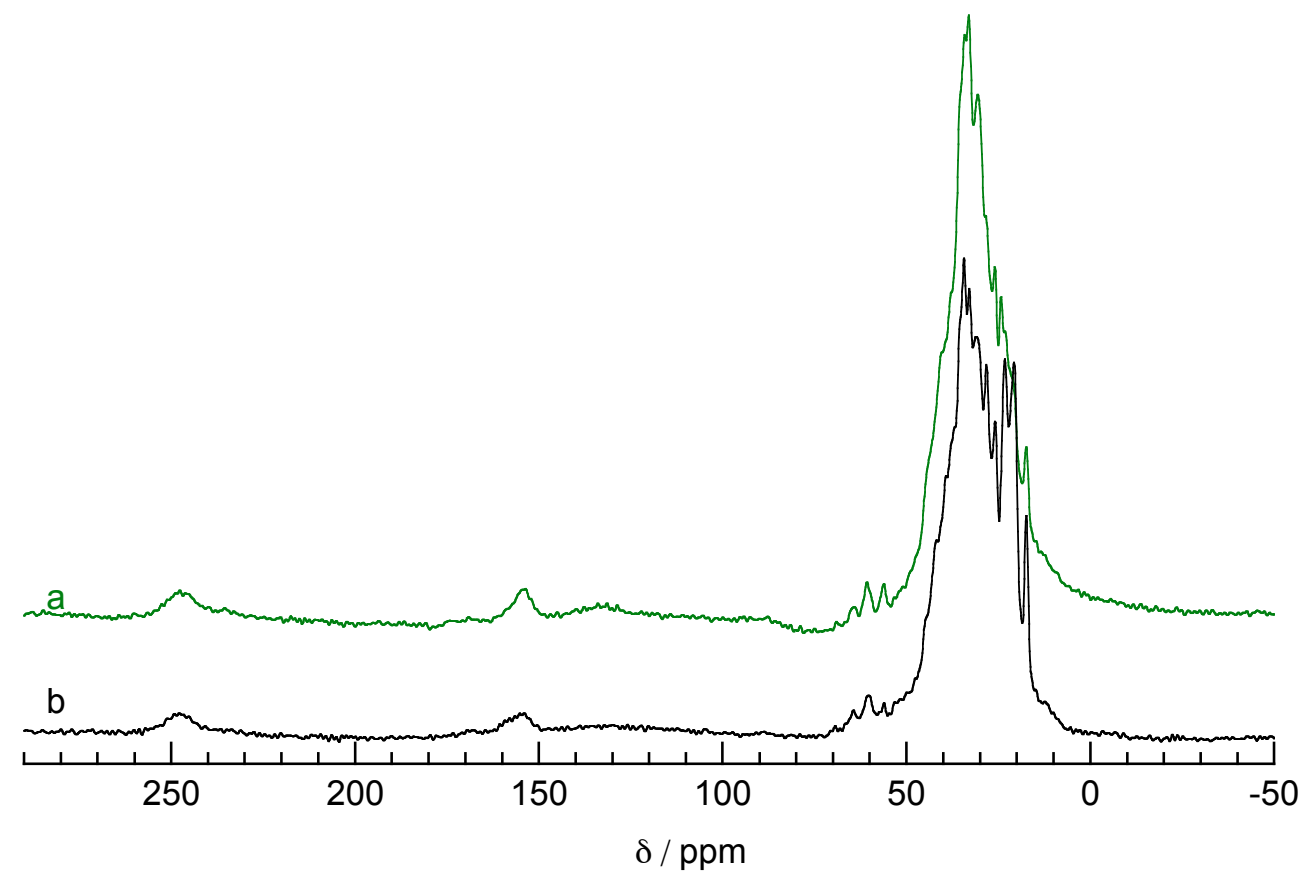

Figure S16. Comparison of ${ }^{13} \mathrm{C}$ CP-MAS NMR for (a) $\mathrm{MTO} / \mathrm{Cl}-\mathrm{Al}_{2} \mathrm{O}_{3}(2.4 \mathrm{wt} \% \mathrm{Re}, 4.0 \mathrm{wt} \% \mathrm{Cl}$, green), and (b) $\mathrm{Cl}-\mathrm{Al}_{2} \mathrm{O}_{3}(4.0 \mathrm{wt} \% \mathrm{Cl}$, black), after $1 \mathrm{~h}$ reaction of $250 \mathrm{mg}$ solid with 300 Torr $\mathrm{CH}_{3}{ }^{13} \mathrm{CH}=\mathrm{CH}_{2}$ at $0{ }^{\circ} \mathrm{C}$, followed by evacuation overnight $\left(10^{-4}\right.$ Torr $)$ at $\mathrm{RT}$. In addition to a broad, complex feature from $20-60 \mathrm{ppm}$, due to $s p^{3}$-hybridized carbon of adsorbed olefins, the weak signals at 155 and $246 \mathrm{ppm}$ are assigned to $s p^{2}$-hybridized carbon of adsorbed olefins, and adsorbed cationic species, respectively. ${ }^{5}$ 

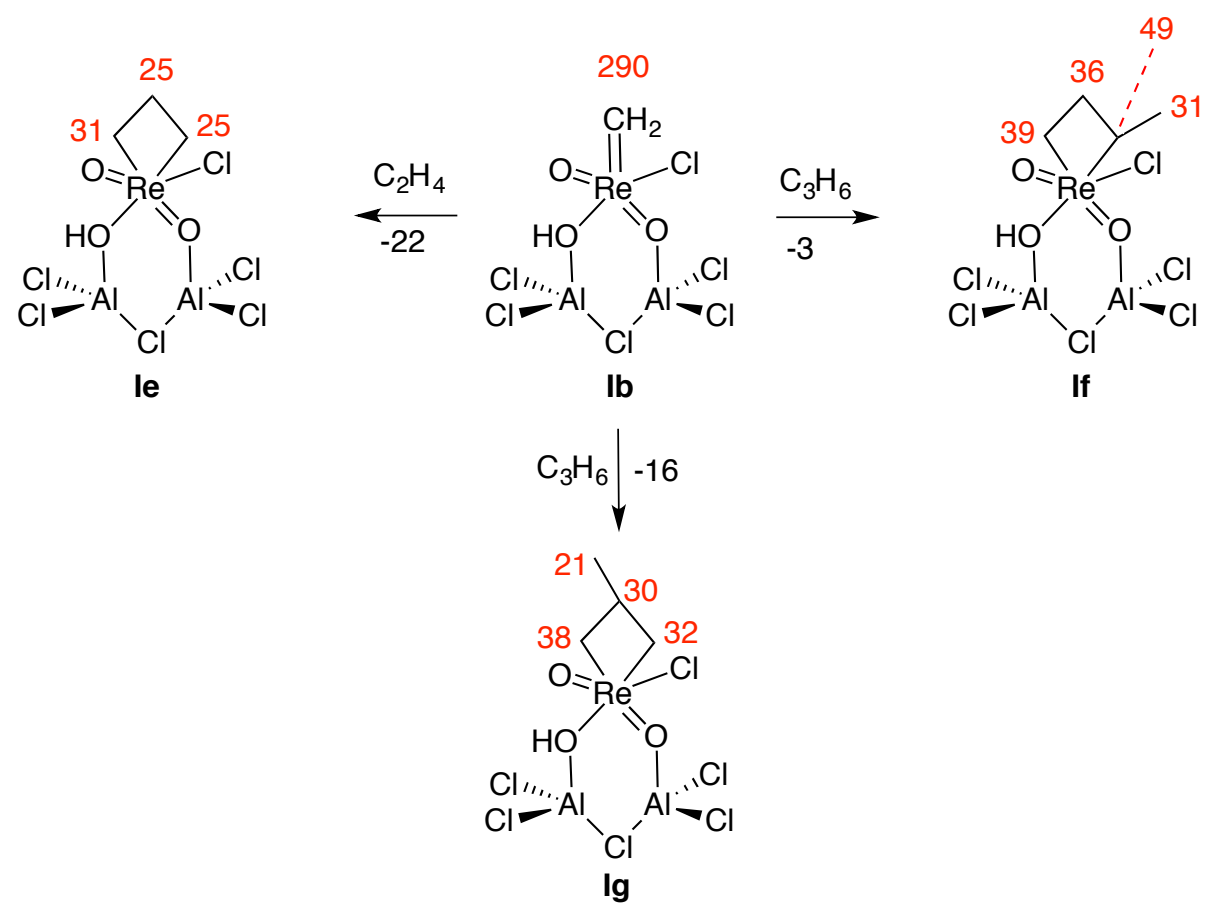

Scheme S4. DFT-optimized energies (in black) and computed ${ }^{13} \mathrm{C}$ NMR chemical shifts (in red) for rhenacyclobutanes derived from a terminal carbene.

Table S5. Calculated ${ }^{13} \mathrm{C}$ chemical shifts (ppm) for molecular organorhenium(VII) compounds ${ }^{6}$

\begin{tabular}{lcccc}
\hline compound & solvent & $\begin{array}{c}\text { Calculated } \\
\text { isotropic } \\
\text { shielding }\end{array}$ & $\begin{array}{c}\text { Calculated } \\
\text { chemical } \\
\text { shift }\end{array}$ & $\begin{array}{c}\text { Observed } \\
\text { chemical shift }\end{array}$ \\
\hline $\mathrm{CH}_{3} \mathrm{ReO}_{3}$ & $\mathrm{CDCl}_{3}$ & 173.5 & 17.7 & 19.6 \\
& $\mathrm{C}_{6} \mathrm{H}_{6}$ & 175.6 & 15.8 & 18.1 \\
$\left(\mathrm{Me}_{3} \mathrm{CCH}_{2}\right)_{2} \mathrm{PhReO}_{2}$ & $\mathrm{CDCl}_{3}$ & 55.7 & 129.2 & $131.0(\mathrm{~m}-\mathrm{C})$ \\
& & 54.5 & 130.4 & $130.5(o-\mathrm{C})$ \\
& & 60.7 & 124.5 & $124.1(p-\mathrm{C})$ \\
& & 115.1 & 73.0 & $75.9\left(\mathrm{CH}_{2}\right)$ \\
& & 153.4 & 36.8 & $36.4\left(\mathrm{CH}_{2} \mathrm{C}\right)$ \\
& & 157.8 & 32.6 & $31.8\left(\mathrm{CH}_{3}\right)$ \\
$\mathrm{Me}_{3} \mathrm{CCH}_{2} \mathrm{ReO}_{2}\left(=\mathrm{CHC}\left(\mathrm{CH}_{3}\right)_{3}\right)$ & $\mathrm{CD}_{3} \mathrm{C}_{6} \mathrm{D}_{5}$ & -107.6 & 283.9 & $283.3\left(\mathrm{CH}_{3}\right)$ \\
& & 142.4 & 47.7 & $45.3\left(\mathrm{CHC}^{6}\right)$ \\
& & 149.6 & 33.4 & $38.5\left(\mathrm{CH}_{2}\right)$ \\
& & 157.0 & 32.6 & $32.1\left(\mathrm{CH}_{3}\right)$ \\
& & 157.8 & 30.5 & $31.3\left(\mathrm{CH}_{2} \mathrm{C}\right)$ \\
& & 160.1 & 30.5 & $29.9\left(\mathrm{CH}_{3}\right)$ \\
\hline
\end{tabular}




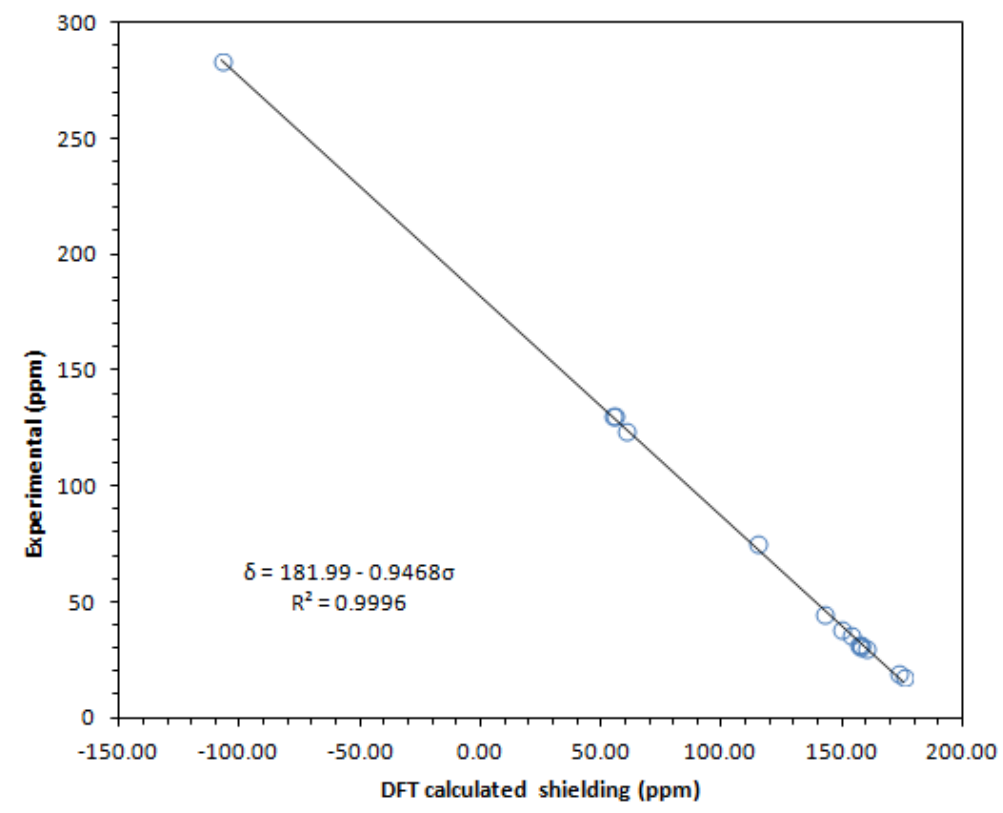

Figure S17. Calibration curve for calculated ${ }^{13} \mathrm{C}$ NMR chemical shifts in organorhenium(VII) compounds, based on data in Table S5.

Table S6. Calculated ${ }^{1} \mathrm{H}$ chemical shifts (ppm) for molecular organorhenium(VII) compounds ${ }^{6}$

\begin{tabular}{lcccc}
\hline Compound & Solvent & $\begin{array}{c}\text { Observed } \\
\text { chemical } \\
\text { shift }\end{array}$ & $\begin{array}{c}\text { Calculated } \\
\text { isotropic } \\
\text { shielding }\end{array}$ & $\begin{array}{c}\text { Calculated } \\
\text { chemical } \\
\text { shift }\end{array}$ \\
\hline $\mathrm{CH}_{3} \mathrm{ReO}_{3}$ & $\mathrm{CDCl}_{3}$ & 2.64 & 30.30 & 2.15 \\
$\left(\mathrm{Me}_{3} \mathrm{CCH}_{2}\right)_{2} \mathrm{PhReO}_{2}$ & $\mathrm{C}_{6} \mathrm{H}_{6}$ & 1.25 & 30.40 & 2.07 \\
& $\mathrm{CD}_{3} \mathrm{CN}_{3}$ & $7.30(m-\mathrm{H})$ & 24.29 & 7.19 \\
& & $7.05(o-\mathrm{H})$ & 24.60 & 6.94 \\
& & $6.97(p-\mathrm{H})$ & 24.54 & 6.98 \\
& & $3.42\left(\mathrm{CH}_{2}\right)$ & 29.53 & 2.79 \\
$\mathrm{Me}_{3} \mathrm{CCH}_{2} \mathrm{ReO}_{2}\left(=\mathrm{CHC}\left(\mathrm{CH}_{3}\right)_{3}\right)$ & $\mathrm{CD}_{3} \mathrm{C}_{6} \mathrm{H}_{6}$ & $1.07\left(\mathrm{CH}_{3}\right)$ & 30.94 & 1.61 \\
& & $2.07\left(\mathrm{CH}^{2}\right)$ & 18.23 & 12.32 \\
& & $2.63\left(\mathrm{CH}_{2}\right)$ & 29.96 & 2.44 \\
& & $0.95\left(\mathrm{CH}_{3}\right)$ & 30.32 & 2.13 \\
& & & 30.87 & 1.68 \\
\hline
\end{tabular}




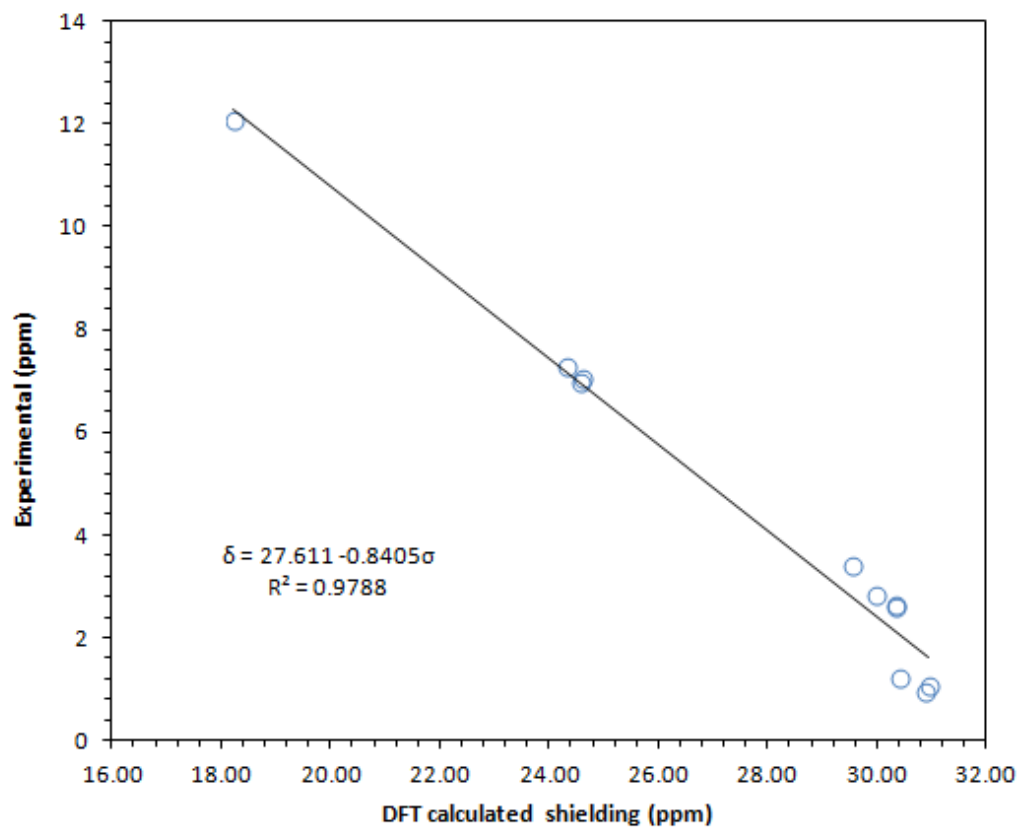

Figure S18. Calibration curve for calculated ${ }^{1} \mathrm{H}$ NMR chemical shifts in $\operatorname{Re}(\mathrm{VII})$ compounds, based on data in Table S6.

Table S7. DFT-optimized bond lengths ( $\AA$ ) for carbene tautomers of MTO interacting with $\mathrm{Al}_{2} \mathrm{Cl}_{6}$

\begin{tabular}{lccccccc}
\hline & Ib & Ic & Id & IIb & IIc & IIIb & Vb \\
\hline Re-O1 & 2.18 & 2.20 & 1.87 & 1.91 & 2.01 & 1.76 & 1.77 \\
Re-O2 & 1.80 & 1.67 & 1.75 & 2.05 & 1.65 & 1.67 & 1.66 \\
Re-O3 & 1.68 & 1.67 & 1.87 & 1.66 & 1.89 & 1.87 & 1.86 \\
Re-Cl & 2.29 & 2.27 & 2.33 & 2.30 & 2.40 & -- & 3.56 \\
Re-C & 1.86 & 2.02 & 1.84 & 1.85 & 1.88 & 1.86 & 1.85 \\
\hline
\end{tabular}


Table S8. Cartesian coordinates and total energies (TE, in Hartrees) for all computed structures

\begin{tabular}{lrrr}
\multicolumn{4}{l}{ Ia: $\mathrm{TE}=-3590.63486668$} \\
$\mathrm{O}$ & -1.651907 & 0.556115 & 1.919167 \\
$\mathrm{Re}$ & -2.660458 & 1.990615 & 1.614615 \\
$\mathrm{O}$ & -1.271685 & 3.106268 & 1.691572 \\
$\mathrm{C}$ & -3.301477 & 0.837320 & -0.065137 \\
$\mathrm{O}$ & -3.760202 & 1.908072 & 2.859489 \\
$\mathrm{H}$ & -4.297846 & 1.121836 & -0.387367 \\
$\mathrm{H}$ & -2.584681 & 1.059044 & -0.860719 \\
$\mathrm{H}$ & -3.240591 & -0.218961 & 0.184491 \\
$\mathrm{Cl}$ & -3.774872 & 3.616337 & 0.406602 \\
$\mathrm{Cl}$ & 1.357597 & 1.426896 & 1.688320 \\
$\mathrm{Cl}$ & 1.195090 & 4.720878 & 0.401073 \\
$\mathrm{Al}$ & -0.094274 & -0.351992 & 1.690382 \\
$\mathrm{Al}$ & 0.453633 & 3.546460 & 1.967863 \\
$\mathrm{Cl}$ & 0.280800 & -1.428100 & 3.470400 \\
$\mathrm{Cl}$ & 0.754000 & 4.068500 & 3.975400 \\
$\mathrm{Cl}$ & -0.103100 & -1.042900 & -0.320700
\end{tabular}

$\begin{array}{lrrr}\text { IIIa: } & \text { TE }=-3590.62699217 & \\ \mathrm{O} & -1.783235 & 4.148541 & 2.510894 \\ \mathrm{Re} & -2.714442 & 5.607050 & 2.540048 \\ \mathrm{O} & -4.366174 & 5.354802 & 2.376622 \\ \mathrm{C} & -2.449484 & 6.363684 & 4.444743 \\ \mathrm{O} & -2.167808 & 6.814601 & 1.515633 \\ \mathrm{H} & -2.963031 & 7.320581 & 4.548000 \\ \mathrm{H} & -1.374368 & 6.489708 & 4.596598 \\ \mathrm{H} & -2.843338 & 5.642610 & 5.163769 \\ \mathrm{Cl} & -1.473867 & 1.311702 & 2.109230 \\ \mathrm{Cl} & 1.673255 & 1.368890 & 1.840316 \\ \mathrm{Cl} & 0.260021 & 4.063289 & 0.294943 \\ \mathrm{Al} & 0.160086 & -0.269140 & 1.674564 \\ \mathrm{Al} & -0.056016 & 3.162342 & 2.189375 \\ \mathrm{Cl} & 0.280800 & -1.428100 & 3.470400 \\ \mathrm{Cl} & 0.754000 & 4.068500 & 3.975400 \\ \mathrm{Cl} & -0.103100 & -1.042900 & -0.320700\end{array}$

IIa: $\mathrm{TE}=-3590.62632943$

$\begin{array}{lrrr}\mathrm{O} & -0.912618 & 1.206574 & 2.074639 \\ \mathrm{Re} & -2.845675 & 1.106552 & 1.920955 \\ \mathrm{O} & -3.118549 & -0.426592 & 2.525953 \\ \mathrm{C} & -4.773934 & 1.788555 & 2.528282 \\ \mathrm{O} & -3.170795 & 1.164838 & 0.290382 \\ \mathrm{H} & -4.981368 & 2.776744 & 2.127569 \\ \mathrm{H} & -4.852362 & 1.792709 & 3.613239 \\ \mathrm{H} & -5.453756 & 1.048392 & 2.101197 \\ \mathrm{Cl} & -2.400080 & 3.160667 & 3.088826 \\ \mathrm{Cl} & 1.863508 & 1.309577 & 1.865640 \\ \mathrm{Cl} & -0.142990 & 3.856551 & 0.470960 \\ \mathrm{Al} & 0.248578 & -0.245541 & 1.675339 \\ \mathrm{Al} & 0.069799 & 2.862006 & 2.327665 \\ \mathrm{Cl} & 0.280800 & -1.428100 & 3.470400 \\ \mathrm{Cl} & 0.754000 & 4.068500 & 3.975400 \\ \mathrm{Cl} & -0.103100 & -1.042900 & -0.320700\end{array}$

IVa: $\mathrm{TE}=-3590.61609548$

$\begin{array}{lrrr}\mathrm{O} & 0.225901 & 3.794686 & 0.618558 \\ \mathrm{Re} & 1.113095 & 4.902381 & -0.561722 \\ \mathrm{O} & 0.153233 & 6.271345 & -0.608331 \\ \mathrm{C} & 2.880211 & 5.990476 & -1.067929 \\ \mathrm{O} & 1.042855 & 4.095115 & -2.023018 \\ \mathrm{H} & 2.609937 & 6.508456 & -1.990348 \\ \mathrm{H} & 3.133679 & 6.714533 & -0.296647 \\ \mathrm{H} & 3.722781 & 5.326878 & -1.247205 \\ \mathrm{Cl} & -1.088087 & 1.534957 & 2.133288 \\ \mathrm{Cl} & 2.023368 & 1.120254 & 1.744566 \\ \mathrm{Cl} & 2.777047 & 4.316386 & 1.197304 \\ \mathrm{Al} & 0.171639 & -0.281308 & 1.675489 \\ \mathrm{Al} & 0.807973 & 3.051915 & 2.110087 \\ \mathrm{Cl} & 0.280800 & -1.428100 & 3.470400 \\ \mathrm{Cl} & 0.754000 & 4.068500 & 3.975400 \\ \mathrm{Cl} & -0.103100 & -1.042900 & -0.320700\end{array}$


Va: $\mathrm{TE}=-3590.63859580$

$\begin{array}{lrcc}\mathrm{O} & -0.603636 & 3.518647 & 1.098635 \\ \mathrm{Re} & -2.071978 & 3.260224 & 0.160183 \\ \mathrm{O} & -3.553999 & 3.427676 & 0.921381 \\ \mathrm{C} & -2.035991 & 4.963658 & -1.010653 \\ \mathrm{O} & -2.086190 & 2.074840 & -1.018771 \\ \mathrm{H} & -2.883005 & 4.962028 & -1.696893 \\ \mathrm{H} & -2.084906 & 5.832455 & -0.351667 \\ \mathrm{H} & -1.097445 & 4.965443 & -1.569010 \\ \mathrm{Cl} & -1.978345 & 0.829175 & 2.015951 \\ \mathrm{Cl} & 1.548294 & 1.313199 & 1.724275 \\ \mathrm{Cl} & 2.293192 & 4.608334 & 0.733875 \\ \mathrm{Al} & -0.169691 & -0.339486 & 1.699843 \\ \mathrm{Al} & 1.011503 & 3.485409 & 1.974648 \\ \mathrm{Cl} & 0.280800 & -1.428100 & 3.470400 \\ \mathrm{Cl} & 0.754000 & 4.068500 & 3.975400 \\ \mathrm{Cl} & -0.103100 & -1.042900 & -0.320700\end{array}$

Ib: $\mathrm{TE}=-3590.63758034$

$\begin{array}{lccc}\mathrm{O} & -1.416029 & 0.743726 & 2.012115 \\ \mathrm{Re} & -2.348196 & 2.426951 & 0.978669 \\ \mathrm{O} & -0.689769 & 3.085587 & 1.215733 \\ \mathrm{C} & -2.445507 & 1.566465 & -0.663283 \\ \mathrm{O} & -3.687798 & 1.926276 & 1.851349 \\ \mathrm{H} & -3.266146 & 0.918907 & -0.956623 \\ \mathrm{H} & -1.629835 & 1.679976 & -1.375768 \\ \mathrm{H} & -1.941611 & 0.468466 & 2.769117 \\ \mathrm{Cl} & -3.169215 & 4.483052 & 0.379981 \\ \mathrm{Cl} & 1.724782 & 1.266310 & 1.767378 \\ \mathrm{Cl} & 2.110963 & 4.567576 & 0.659033 \\ \mathrm{Al} & 0.043448 & -0.277778 & 1.692122 \\ \mathrm{Al} & 0.898707 & 3.447224 & 1.958696 \\ \mathrm{Cl} & 0.280800 & -1.428100 & 3.470400 \\ \mathrm{Cl} & 0.754000 & 4.068500 & 3.975400 \\ \mathrm{Cl} & -0.103100 & -1.042900 & -0.320700\end{array}$

IIb: TE $=-3590.62852904$

$\begin{array}{llll}\mathrm{O} & -0.992356 & 1.421810 & 2.548853\end{array}$

$\begin{array}{llll}\text { Re } & -2.792255 & 1.038371 & 3.066072\end{array}$

$\begin{array}{llll}\mathrm{O} & -4.307122 & 1.048557 & 2.395496\end{array}$

$\begin{array}{llll}\text { C } & -2.949800 & 0.034821 & 4.614495\end{array}$

$\begin{array}{llll}\mathrm{O} & -2.143236 & -0.449303 & 1.821582\end{array}$

$\mathrm{H} \quad-3.880540 \quad-0.372860 \quad 4.997484$

$\mathrm{H} \quad-2.035426 \quad-0.193913 \quad 5.166294$

$\mathrm{H} \quad \begin{array}{llll}-2.595900 & -1.288268 & 1.713532\end{array}$

$\begin{array}{llll}\mathrm{Cl} & -2.834745 & 3.131518 & 4.013532\end{array}$

$\begin{array}{llll}\mathrm{Cl} & 1.626680 & 1.309809 & 1.635489\end{array}$

$\begin{array}{llll}\mathrm{Cl} & -0.623242 & 3.928899 & 0.666076\end{array}$

$\begin{array}{llll}\mathrm{Al} & -0.241860 & -0.257626 & 1.704552\end{array}$

$\begin{array}{llll}\text { Al } & 0.156588 & 2.870927 & 2.320316\end{array}$

$\begin{array}{llll}\mathrm{Cl} & 0.280795 & -1.428083 & 3.470345\end{array}$

$\begin{array}{llll}\mathrm{Cl} & 0.753999 & 4.068488 & 3.975398\end{array}$

$\begin{array}{lllll}\mathrm{Cl} & -0.103094 & -1.042905 & -0.320643\end{array}$
IIIb: TE $=-3590.60037722$

$\begin{array}{lrrr}\mathrm{O} & -1.842881 & 4.077272 & 2.558308 \\ \mathrm{Re} & -2.319694 & 5.610546 & 3.271228 \\ \mathrm{O} & -2.237723 & 5.481140 & 5.129593 \\ \mathrm{C} & -1.098646 & 6.901102 & 2.739144 \\ \mathrm{O} & -3.803735 & 6.229723 & 2.831687 \\ \mathrm{H} & -1.361900 & 7.890148 & 2.377356 \\ \mathrm{H} & -0.046999 & 6.616552 & 2.689200 \\ \mathrm{H} & -1.481245 & 5.787376 & 5.636985 \\ \mathrm{Cl} & -1.527329 & 1.271306 & 2.091885 \\ \mathrm{Cl} & 1.615950 & 1.398509 & 1.840087 \\ \mathrm{Cl} & 0.226358 & 4.142891 & 0.356708 \\ \mathrm{Al} & 0.139819 & -0.266960 & 1.675592 \\ \mathrm{Al} & -0.178955 & 3.168464 & 2.203483 \\ \mathrm{Cl} & 0.280800 & -1.428100 & 3.470400 \\ \mathrm{Cl} & 0.754000 & 4.068500 & 3.975400 \\ \mathrm{Cl} & -0.103100 & -1.042900 & -0.320700\end{array}$


Vb: $\mathrm{TE}=-3590.61729006$

$\begin{array}{lccc}\mathrm{O} & -0.429272 & 3.706750 & 1.011453 \\ \mathrm{Re} & -1.730816 & 3.681085 & -0.192057 \\ \mathrm{O} & -1.850290 & 4.976763 & -1.225193 \\ \mathrm{C} & -1.455004 & 2.253832 & -1.338876 \\ \mathrm{O} & -3.373869 & 3.436170 & 0.636303 \\ \mathrm{H} & -3.779730 & 2.567896 & 0.733369 \\ \mathrm{H} & -1.525926 & 2.326849 & -2.420145 \\ \mathrm{H} & -1.131911 & 1.291725 & -0.933722 \\ \mathrm{Cl} & -1.964867 & 0.849639 & 1.953754 \\ \mathrm{Cl} & 1.539046 & 1.315389 & 1.687604 \\ \mathrm{Cl} & 2.629385 & 4.510459 & 0.895987 \\ \mathrm{Al} & -0.154923 & -0.307553 & 1.718506 \\ \mathrm{Al} & 1.102437 & 3.538016 & 1.972037 \\ \mathrm{Cl} & 0.280800 & -1.428100 & 3.470400 \\ \mathrm{Cl} & 0.754000 & 4.068500 & 3.975400 \\ \mathrm{Cl} & -0.103100 & -1.042900 & -0.320700\end{array}$

Ic: $\mathrm{TE}=-3590.59616051$

$\begin{array}{lrrr}\mathrm{O} & -4.064866 & 3.182911 & 0.897892 \\ \mathrm{Re} & -3.100601 & 1.859898 & 1.223415 \\ \mathrm{O} & -1.935155 & 0.179542 & 2.028609 \\ \mathrm{C} & -2.070066 & 2.788080 & 2.693767 \\ \mathrm{O} & -2.140572 & 1.682005 & -0.130378 \\ \mathrm{H} & -2.610503 & 3.670249 & 3.039907 \\ \mathrm{H} & -1.873405 & 2.086450 & 3.506573 \\ \mathrm{H} & -2.342252 & -0.319636 & 2.742845 \\ \mathrm{Cl} & -4.775749 & 0.384275 & 1.624390 \\ \mathrm{Cl} & 0.970625 & 1.545563 & 1.635762 \\ \mathrm{Cl} & -0.207324 & 4.714239 & 0.518513 \\ \mathrm{Al} & -0.228070 & -0.376676 & 1.681275 \\ \mathrm{Al} & -0.218205 & 3.453062 & 2.210807 \\ \mathrm{Cl} & 0.280796 & -1.428079 & 3.470345 \\ \mathrm{Cl} & 0.753996 & 4.068468 & 3.975384 \\ \mathrm{Cl} & -0.103091 & -1.042889 & -0.320630\end{array}$

IIc: $\mathrm{TE}=-3590.60712218$

$\begin{array}{lrrr}\mathrm{O} & -1.006262 & 1.158371 & 2.024537 \\ \mathrm{Re} & -2.991088 & 1.413568 & 1.828657 \\ \mathrm{O} & -3.926337 & 0.280398 & 2.577062 \\ \mathrm{C} & -4.233206 & 2.701106 & 1.265194 \\ \mathrm{O} & -2.717768 & 0.817351 & 0.060805 \\ \mathrm{H} & -3.918883 & 3.624332 & 0.779592 \\ \mathrm{H} & -5.294588 & 2.615500 & 1.486509 \\ \mathrm{H} & -1.941055 & 0.262002 & -0.116647 \\ \mathrm{Cl} & -2.375472 & 2.893773 & 3.614482 \\ \mathrm{Cl} & 1.765006 & 1.390838 & 1.846332 \\ \mathrm{Cl} & -0.694654 & 3.886919 & 0.706664 \\ \mathrm{Al} & 0.174485 & -0.207802 & 1.705707 \\ \mathrm{Al} & -0.093728 & 2.794292 & 2.456603 \\ \mathrm{Cl} & 0.280800 & -1.428100 & 3.470400 \\ \mathrm{Cl} & 0.754000 & 4.068500 & 3.975400 \\ \mathrm{Cl} & -0.103100 & -1.042900 & -0.320700\end{array}$

Id: TE $=\mathbf{- 3 5 9 0 . 6 0 2 5 4 2 6 6}$

$\begin{array}{lccc}\mathrm{O} & -1.418504 & 0.801826 & 1.907176 \\ \mathrm{Re} & -2.209797 & 2.269955 & 1.072495 \\ \mathrm{O} & -0.735348 & 3.199200 & 1.243181 \\ \mathrm{C} & -2.206609 & 1.778629 & -0.702622 \\ \mathrm{O} & -3.757999 & 1.565975 & 1.837882 \\ \mathrm{H} & -2.983140 & 1.130082 & -1.101446 \\ \mathrm{H} & -1.406047 & 2.086029 & -1.370070 \\ \mathrm{H} & -3.637414 & 0.800743 & 2.411510 \\ \mathrm{Cl} & -3.510563 & 4.192974 & 0.910464 \\ \mathrm{Cl} & 1.664178 & 1.315554 & 1.761051 \\ \mathrm{Cl} & 2.046019 & 4.614965 & 0.609151 \\ \mathrm{Al} & -0.060547 & -0.296800 & 1.705648 \\ \mathrm{Al} & 0.936967 & 3.466462 & 1.968548 \\ \mathrm{Cl} & 0.280800 & -1.428100 & 3.470400 \\ \mathrm{Cl} & 0.754000 & 4.068500 & 3.975400 \\ \mathrm{Cl} & -0.103100 & -1.042900 & -0.320700\end{array}$




\begin{tabular}{lrrr}
\multicolumn{4}{l}{ Ie: $\mathrm{TE}=-3669.25400016$} \\
$\mathrm{H}$ & -3.943831 & 2.052437 & -1.942953 \\
$\mathrm{H}$ & -3.173774 & 3.937858 & -0.559831 \\
$\mathrm{H}$ & -4.724948 & 3.741183 & 0.291177 \\
$\mathrm{H}$ & -5.002982 & 1.486320 & -0.636986 \\
$\mathrm{C}$ & -3.845354 & 3.224668 & -0.079782 \\
$\mathrm{C}$ & -4.043419 & 1.949380 & -0.862164 \\
$\mathrm{O}$ & -1.564754 & 0.614149 & 2.066409 \\
$\mathrm{Re}$ & -2.657266 & 2.460495 & 1.584814 \\
$\mathrm{O}$ & -0.948150 & 3.009636 & 1.424862 \\
$\mathrm{C}$ & -2.910378 & 1.234952 & -0.180922 \\
$\mathrm{O}$ & -3.777521 & 1.740029 & 2.600958 \\
$\mathrm{H}$ & -3.060991 & 0.209343 & 0.142260 \\
$\mathrm{H}$ & -1.950078 & 1.325082 & -0.690731 \\
$\mathrm{H}$ & -2.078118 & 0.164874 & 2.746724 \\
$\mathrm{Cl}$ & -3.228461 & 4.607407 & 2.237932 \\
$\mathrm{Cl}$ & 1.603262 & 1.311422 & 1.743389 \\
$\mathrm{Cl}$ & 1.686419 & 4.598613 & 0.504554 \\
$\mathrm{Al}$ & -0.025309 & -0.280431 & 1.690718 \\
$\mathrm{Al}$ & 0.700807 & 3.444580 & 1.959100 \\
$\mathrm{Cl}$ & 0.280800 & -1.428100 & 3.470400 \\
$\mathrm{Cl}$ & 0.754000 & 4.068500 & 3.975400 \\
$\mathrm{Cl}$ & -0.103100 & -1.042900 & -0.320700 \\
& & &
\end{tabular}

If: $\mathrm{TE}=-3708.56864939$

$\begin{array}{lrrr}\mathrm{H} & -3.239618 & 3.993425 & -0.501680 \\ \mathrm{H} & -4.883586 & 1.447373 & -0.779078 \\ \mathrm{H} & -3.819560 & 2.160894 & -2.003258 \\ \mathrm{H} & -5.797815 & 3.957406 & -0.752167 \\ \mathrm{H} & -5.247962 & 4.799222 & 0.702823 \\ \mathrm{H} & -5.888752 & 3.153697 & 0.815121 \\ \mathrm{C} & -3.924264 & 3.246883 & -0.091849 \\ \mathrm{C} & -3.950389 & 1.990498 & -0.932939 \\ \mathrm{C} & -5.288677 & 3.834099 & 0.209359 \\ \mathrm{O} & -1.585436 & 0.601663 & 2.054798 \\ \mathrm{Re} & -2.680989 & 2.448138 & 1.581494 \\ \mathrm{O} & -0.968079 & 3.005735 & 1.441882 \\ \mathrm{C} & -2.805152 & 1.311215 & -0.244285 \\ \mathrm{O} & -3.831482 & 1.680794 & 2.529066 \\ \mathrm{H} & -2.911721 & 0.260445 & 0.007196 \\ \mathrm{H} & -1.833287 & 1.478272 & -0.710389 \\ \mathrm{H} & -2.114391 & 0.136685 & 2.712184 \\ \mathrm{Cl} & -3.204129 & 4.570901 & 2.332388 \\ \mathrm{Cl} & 1.589710 & 1.313221 & 1.742706\end{array}$

$\begin{array}{lrrc}\mathrm{Cl} & 1.654839 & 4.598327 & 0.497245 \\ \mathrm{Al} & -0.035017 & -0.280429 & 1.691188 \\ \mathrm{Al} & 0.680026 & 3.443527 & 1.960425 \\ \mathrm{Cl} & 0.280800 & -1.428100 & 3.470400 \\ \mathrm{Cl} & 0.754000 & 4.068500 & 3.975400 \\ \mathrm{Cl} & -0.103100 & -1.042900 & -0.320700\end{array}$

\begin{tabular}{lrrr}
\multicolumn{4}{l}{ Ig: TE $=-3708.57409065$} \\
$\mathrm{H}$ & -3.184874 & 3.948637 & -0.543782 \\
$\mathrm{H}$ & -4.733352 & 3.725070 & 0.309579 \\
$\mathrm{H}$ & -3.873143 & 2.088571 & -1.930507 \\
$\mathrm{H}$ & -5.462371 & 0.334142 & -1.128064 \\
$\mathrm{H}$ & -6.204706 & 1.935710 & -1.040987 \\
$\mathrm{H}$ & -5.600562 & 1.161843 & 0.428545 \\
$\mathrm{C}$ & -3.846525 & 3.223116 & -0.068895 \\
$\mathrm{C}$ & -4.050607 & 1.951136 & -0.862006 \\
$\mathrm{C}$ & -5.408772 & 1.307494 & -0.638035 \\
$\mathrm{O}$ & -1.563217 & 0.615141 & 2.068540 \\
$\mathrm{Re}$ & -2.653386 & 2.465798 & 1.594626 \\
$\mathrm{O}$ & -0.944932 & 3.011882 & 1.422792 \\
$\mathrm{C}$ & -2.921300 & 1.237340 & -0.166031 \\
$\mathrm{O}$ & -3.763068 & 1.754693 & 2.629163 \\
$\mathrm{H}$ & -3.093407 & 0.216342 & 0.166196 \\
$\mathrm{H}$ & -1.956573 & 1.305581 & -0.670708 \\
$\mathrm{H}$ & -2.071976 & 0.170491 & 2.755253 \\
$\mathrm{Cl}$ & -3.220021 & 4.617879 & 2.238331 \\
$\mathrm{Cl}$ & 1.604240 & 1.311001 & 1.743506 \\
$\mathrm{Cl}$ & 1.693431 & 4.598827 & 0.506440 \\
$\mathrm{Al}$ & -0.025661 & -0.279982 & 1.690725 \\
$\mathrm{Al}$ & 0.703669 & 3.445333 & 1.958634 \\
$\mathrm{Cl}$ & 0.280801 & -1.428060 & 3.470384 \\
$\mathrm{Cl}$ & 0.754004 & 4.068507 & 3.975435 \\
$\mathrm{Cl}$ & -0.103106 & -1.042948 & -0.320718
\end{tabular}




\section{Sample Gaussian input file}

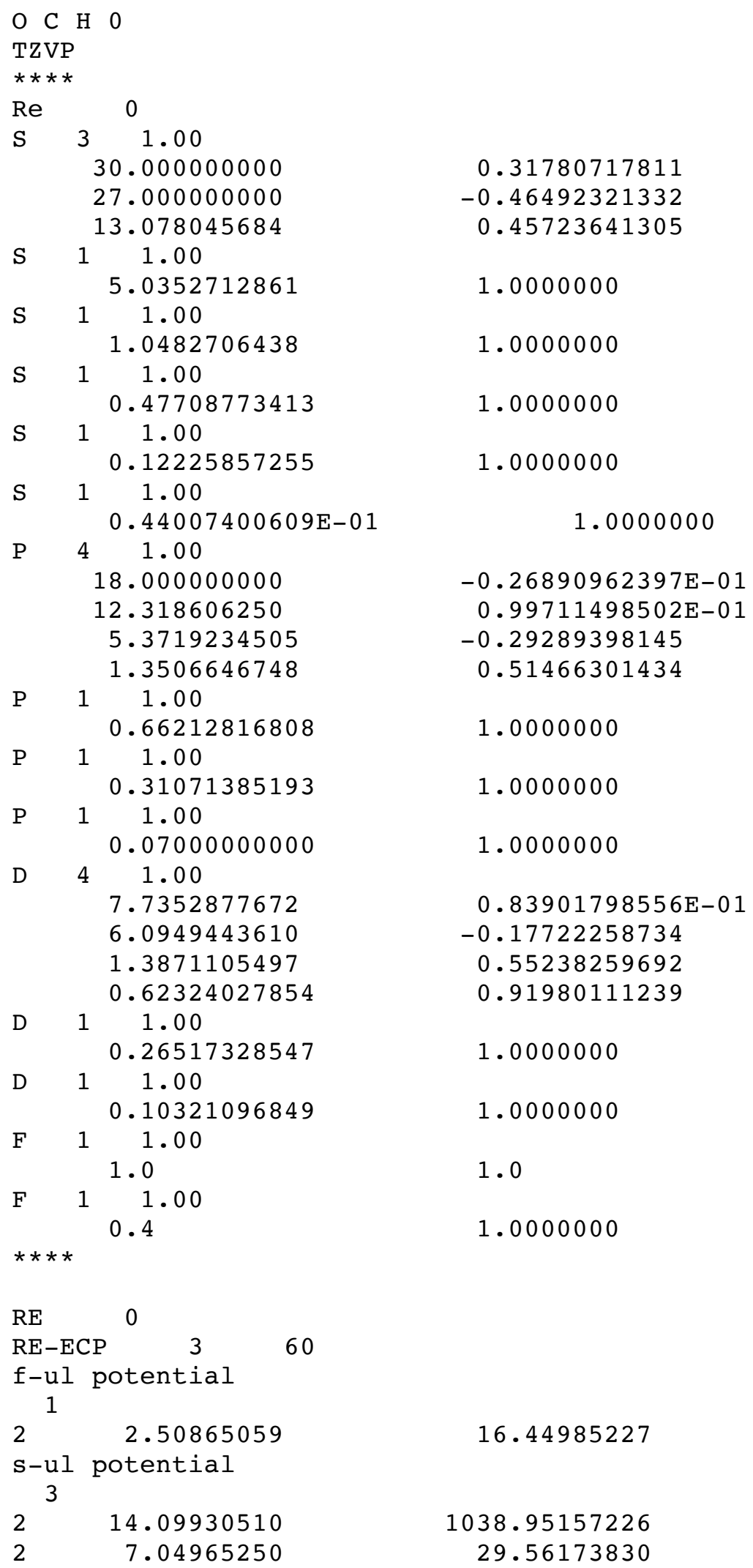




$\begin{array}{lcr}2 & 2.50865059 & -16.44985227 \\ \text { p-ul } & \text { potential } & \\ 3 & & \\ 2 & 10.10771690 & 339.54350965 \\ 2 & 5.05385830 & 24.91369646 \\ 2 & 2.50865059 & -16.44985227 \\ \text { d-ul potential } & \\ 3 & & \\ 2 & 6.84861794 & 111.69965275 \\ 2 & 3.42430897 & 12.62432927 \\ 2 & 2.50865059 & -16.44985227\end{array}$

\section{Additional references}

(1) Parry, E. P. J. Catal. 1963, 2, 371-379.

(2) Salameh, A.; Joubert, J.; Baudouin, A.; Lukens, W.; Delbecq, F.; Sautet, P.; Basset, J. M.; Copéret, C. Angew. Chem., Int. Ed. 2007, 46, 3870-3873.

(3) Tougerti, A.; Cristol, S.; Berrier, E.; Briois, V.; La Fontaine, C.; Villain, F.; Joly, Y. Phys. Rev. B: Condens. Matter Mater. Phys. 2012, 85, 125136.

(4) Fröba, M.; Lochte, K.; Metz, W. J. Phys. Chem. Solids 1996, 57, 635-641.

(5) Haw, J. F.; Richardson, B. R.; Oshiro, I. S.; Lazo, N. D.; Speed, J. A. J. Am. Chem. Soc. 1989, 111, 2052-2058.

(6) Moses, A. W.; Raab, C.; Nelson, R. C.; Leifeste, H. D.; Ramsahye, N. A.; Chattopadhyay, S.; Eckert, J.; Chmelka, B. F.; Scott, S. L. J. Am. Chem. Soc. 2007, 129, 89128920. 